\title{
Dynamic analysis of a bogie for hunting detection through a simplified wheel-rail contact model
}

\author{
M. Bozzone · E. Pennestrì • P. Salvini
}

Received: 17 February 2010 / Accepted: 3 November 2010 / Published online: 7 December 2010

(C) Springer Science+Business Media B.V. 2010

\begin{abstract}
The present paper analyzes the dynamics of a bogie composed of two wheelsets and a frame. The bogie frame is joined to the wheelsets by means of a primary suspension system, acting on the three principal directions, i.e., longitudinal, transverse and vertical.

The bogie moves along rails following its variable path. In particular, the dynamic analysis investigates the bogie behavior in both straight and curved paths, with or without an initial perturbation. Imposing an initial disturbance, the hunting motion is observed and the critical speed value estimated.

The present analysis uses a lookup table for the determination of the contact characteristics. In order to minimize cpu-time, a new procedure for the interpolation of the lookup table entries has been developed. Finally, two different methods for the integration of the differential equations have been tested and comparisons with the results obtained by commercial multibody software are discussed.
\end{abstract}

Keywords Rail dynamics · Hunting speed

\section{Introduction}

The importance of railway networks in the world is increasing together with the requested convoy performances such as speed, load capacity, convoy length. These circumstances require preliminary sophisticated dynamic analyses in order to foresee the behavior of the overall system during normal and transient running, and the identification of potentially critical conditions.

M. Bozzone $\cdot$ E. Pennestrì $(\bowtie) \cdot$ P. Salvini

Department of Mechanical Engineering, University of Rome "Tor Vergata", Via del Politecnico, 1, 00133 Rome, Italy

e-mail: pennestri@mec.uniroma2.it

M. Bozzone

e-mail: michelangelo.bozzone@uniroma2.it

P. Salvini

e-mail: salvini@uniroma2.it 
Train dynamic analyses suffer the peculiarity of being dependent on multiple scale phenomena. In fact, the analyses are based on the simultaneous consideration of the local wheel-rail contact (local scale) and the displacements of the convoy (large scale). Therefore, the multibody analyses of railways systems are forced to handle this circumstance by adopting appropriate concurrent strategies, e.g., [1-4].

This paper can be listed within this research flow, focusing on the search of the hunting phenomena onset. As a matter of fact, although hunting affects large scale dynamics, its origin should be interpreted at a local scale. The reliability of results rests upon an accurate simulation of actual contact conditions.

In a previous paper [5], the authors proposed a new method for detecting single or multiple contacts between wheel and rail. In the present work, the proposed method has been embodied in a large scale dynamic analysis to study the dynamic instability of a bogie while running.

The dynamic analysis of a single wheelset and of an assembled bogie plays an important role for monitoring the different events characterizing the motion. In particular, the following analyses have a significant interest [5-9]:

- Wheelset, bogie and convoy critical speed determination;

- Study of the hunting motion, both in time and frequency domain;

- Analysis of the bogie behavior while turning;

- Analysis of the contact forces;

- Analysis of the limit derailment conditions;

- Study of the forces transferred by the suspension elements to the bogie frame;

- Passenger comfort optimization;

- Study of the acoustic emissions from the contact surfaces.

Rail defects, extraneous bodies on the rails, railroad switches and work imperfections cause sudden perturbations to the motion of a wheelset or of a bogie. Under these conditions, hunting motion can occur with transverse oscillations whose amplitudes, frequencies and eventual decay depend on perturbation itself and on the wheelset velocity [1, 3, 8-10].

The oscillatory motion is caused by the changing of the curvature radii of the wheels in the contact points when the wheelset is displaced from its centered position. In fact, when the barycenter of the wheelset is moved from the middle of the track, the tangent forces acting on the two wheels are different. This unbalance generates a yaw torque. The corresponding yaw motion is coupled with the transverse motion.

The oscillations may affect motion steadiness. In fact, when the bogie has a speed lower than the critical speed, the amplitude of the oscillations decreases with time. On the contrary, when the speed is higher than the critical speed, instability occurs.

Bogies should travel as far as possible from unstable condition because of its influence on contact forces, passenger comfort, noise and the extreme possibility to trig a convoy derailment.

The primary suspension system has a beneficial effect on the stability limits of the bogie. The system acts mainly along the transverse and longitudinal directions. In this manner, the spring-damper suspension elements reduce the hunting motion and, as a consequence, the operating speed can be increased safely.

For these reasons, it is crucial to assess under which operating conditions instability occurs. In particular, an estimate of the critical speed is required to guarantee the system safety. Therefore, in this investigation a software tool for the dynamic analysis of the main bogie components has been developed. A reliable computation of a railway vehicle dynamics needs an accurate wheel-rail contact model. This usually increases the computation time. 
To reduce the cpu-time required by the simulation, the approach of the lookup tables [5] is adopted. In particular, precalculated tables have been compiled in order to evaluate in a very short time the wheel-rail contact characteristics, such as the location of contact points and the direction of normal unit vectors to the surfaces.

The force transmitted between the contact surfaces can be evaluated through different rolling contact theories. A widely used theory in multibody software is the Kalker FASTSIM algorithm [11]. This algorithm is based on the Kalker simplified theory in which the contact surfaces are replaced by a set of springs. The advantages of the method are simplicity of conception and computational speed. FASTSIM is significantly faster than the Kalker CONTACT algorithm which is based on the so called Kalker exact theory. However, the accuracy of results is about $15 \%$ lower than of experimental evidences.

Polach [12] recently developed a method faster than FASTSIM. This method offers a trade-off between calculation time saving and accuracy.

Other appreciably fast methods are those which take advantage of saturations function proposed by Vermeulen [13]. Calculation time is reduced, but significant differences with experimental values are observed when a large spin is present.

FASTSIM is embodied in commercial multibody software such as ADAMS/rail, SIMPACK, MEDYNA, GENSYS, VOCO.

The analysis herein described uses the Kalker linear theory [6, 7]. Tangential forces are linearly dependent on local creepages between the contact surfaces.

The starting point has been the meshing of the wheel and rail and data capture in a Matlab environment. Wheel and rail surfaces are considered as a node grid. The compilation of the lookup tables was obtained by adopting the load center method [5]. The results of this method appear more uniform and less affected by mesh coarseness. In particular, the method uses an iterative search of the intersecting volumes. This is performed by tuning the contact stiffness to the value that guarantees the same compenetration as the one predicted by Hertz. Note that this search solves only the kinematic aspects of contact. The dynamic actual values are computed outside the lookup table during the multibody simulation.

The details of the wheel-rail contact analysis and modeling are discussed in [5].

In order to reduce cpu-time, the standard Matlab function to access and interpolate the numerical values stored in a lookup table has been substituted with a new one. Moreover, two different strategies during the integration of differential equations have been tested; namely the "macrostep method" and "variable step method".

The main components of the bogie are the two wheelsets and a bogie frame. These are connected through a suspension device. This last is modeled by means of spring-damper elements acting towards vertical, longitudinal and transverse directions. The literature records different bogie types and models. The main differences regard the accuracy in modeling the suspension system, the degrees of freedom of the moving masses and the models adopted to manage contact surfaces. Table 1 reports a concise and not exhaustive list of the assumptions made for each model with the appropriate bibliographic references. In the table, $x_{G}, y_{G}, z_{G}$ are the longitudinal, transverse, vertical center of mass position; $\vartheta_{x}, \vartheta_{y}, \vartheta_{z}$ are the roll, pitch and yaw angles.

\section{The bogie modeling}

The bogie examined here is composed of three masses: two wheelsets, a bogie frame and a primary suspension system. The masses, main dimensions and features of the components are summarized in Table 2. 
Table 1 Comparison among different approaches found in the literature

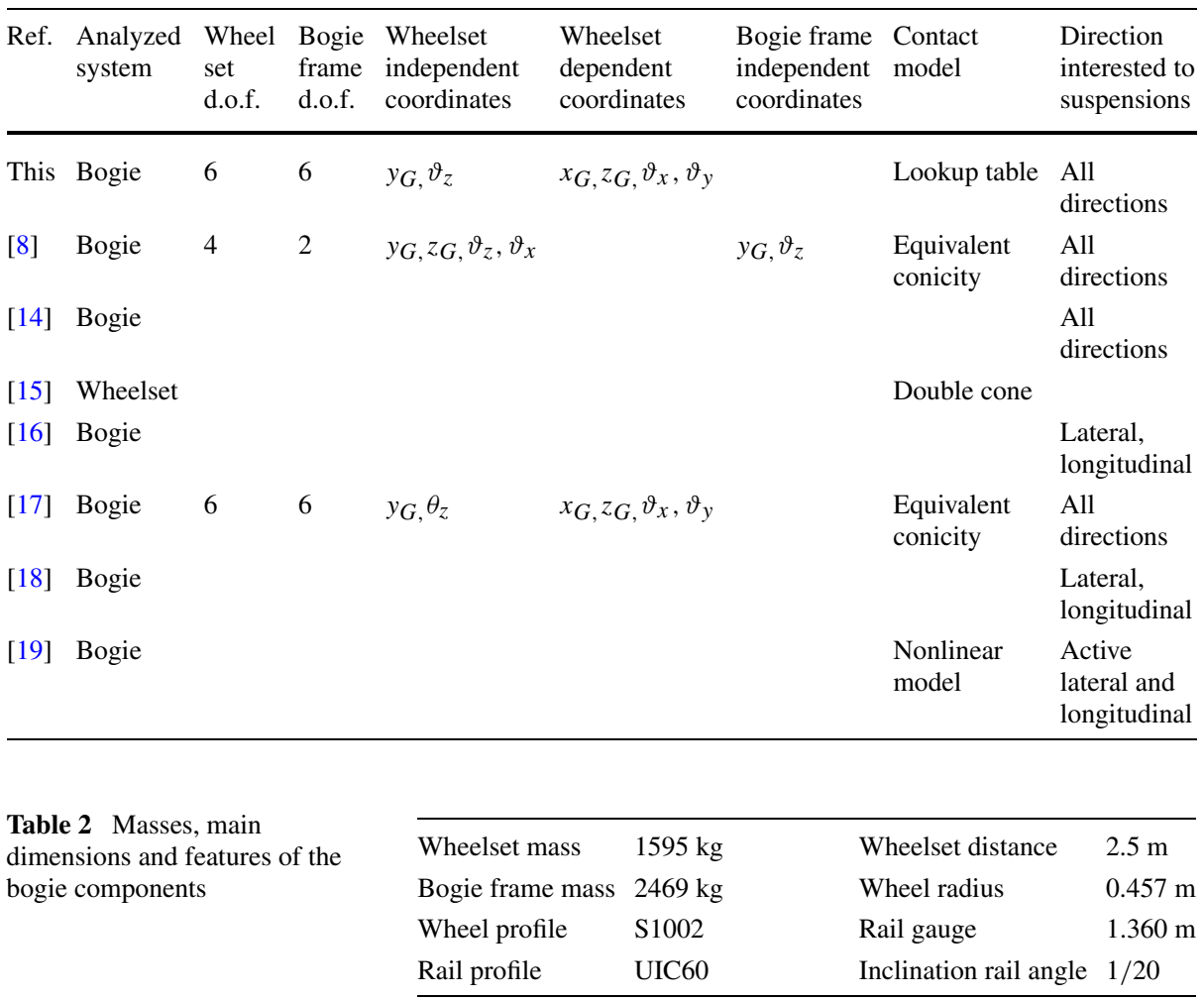

Table 3 Stiffness and damping coefficients of the suspension elements

\begin{tabular}{llll}
\hline Longitudinal spring stiffness & $10^{6} \mathrm{~N} / \mathrm{m}$ & Vertical damping coefficient & $3.04 \cdot 10^{3} \mathrm{Ns} / \mathrm{m}$ \\
Transverse spring stiffness & $2 \cdot 10^{7} \mathrm{~N} / \mathrm{m}$ & Vertical length at rest & $0.5 \mathrm{~m}$ \\
Vertical spring stiffness & $1.5 \cdot 10^{6} \mathrm{~N} / \mathrm{m}$ & Longitudinal length at rest & $0.75 \mathrm{~m}$ \\
Longitudinal damping coefficient & $2.49 \cdot 10^{3} \mathrm{Ns} / \mathrm{m}$ & Transverse length at rest & $0.3 \mathrm{~m}$ \\
Transverse damping coeff. & $1.11 \cdot 10^{4} \mathrm{Ns} / \mathrm{m}$ & &
\end{tabular}

\subsection{The suspension system}

As shown in Fig. 1, for each wheel the primary suspension system has been modeled with three translational spring-damper elements: one for each principal direction. The stiffnesses and the damping of the suspension elements are reported in Table 3.

\section{The wheelset-rail model}

Due to symmetry, we refer only to the front wheelset. The first step in the model generation is the acquisition of wheel and rail profiles (see Fig. 2). The rail surface is obtained by means of an extrusion of the rail profile along the axis of the railway. The wheel surface is 
Fig. 1 The bogie with the primary suspension system together with the reference systems used in the dynamic analysis

Fig. 2 Wheel and rail profile shapes
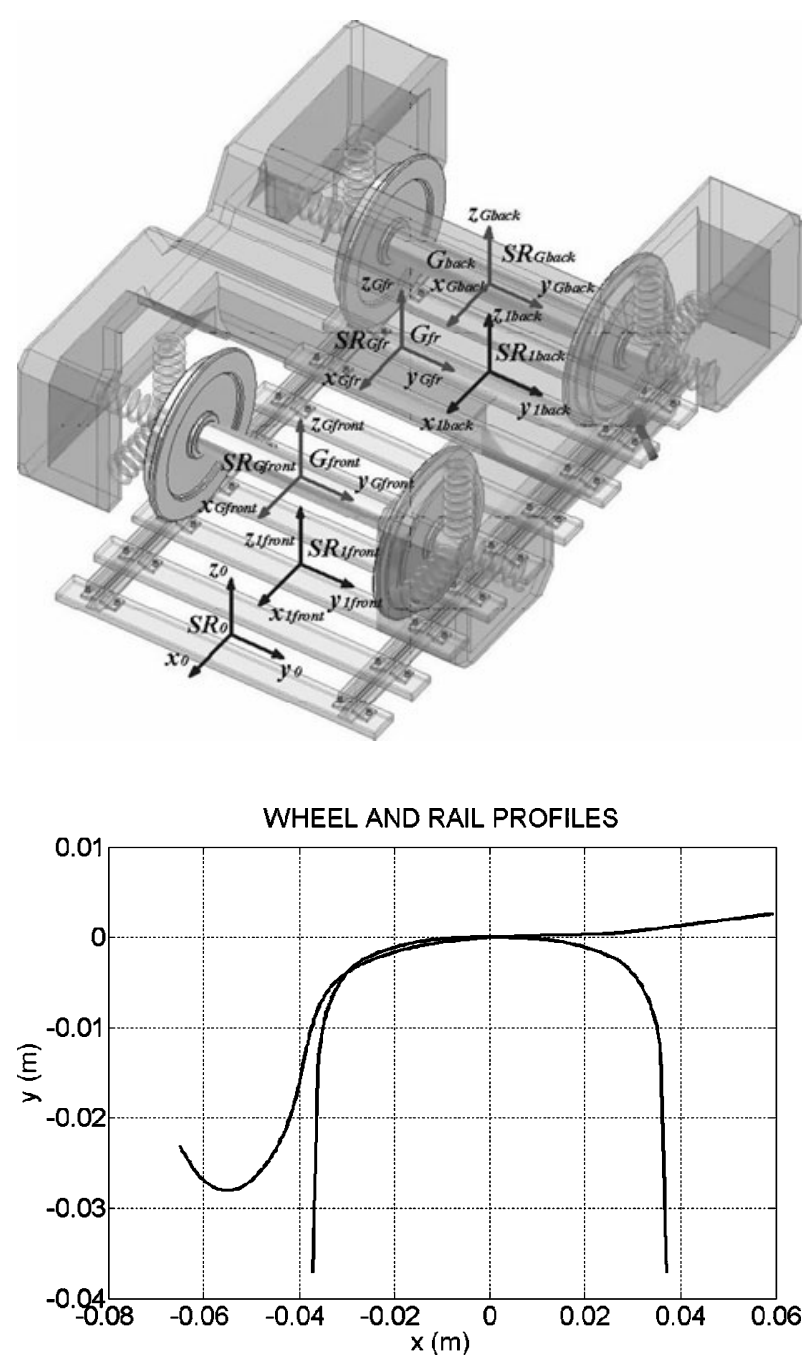

generated through a revolution of the wheel profile around the wheel axis. The two surfaces are accounted by means of a discrete mesh.

\section{The Cartesian coordinate systems}

The following right-hand Cartesian coordinate systems are introduced in the analysis:

- Inertial fixed reference system $S R_{0}$;

- Reference system fixed with the front wheelset $S R_{G \text { front }}$;

- Reference system fixed with the back wheelset $S R_{G \text { back }}$;

- Reference system fixed with the bogie frame $S R_{G \mathrm{fr}}$;

- Reference system that follows the front wheelset on the rail path $S R_{1 \text { front }}$;

- Reference system that follows the back wheelset on the rail path $S R_{1 \text { back }}$. 
Fig. $3 S R_{1}$ attitude in the straight and curved rail path cases

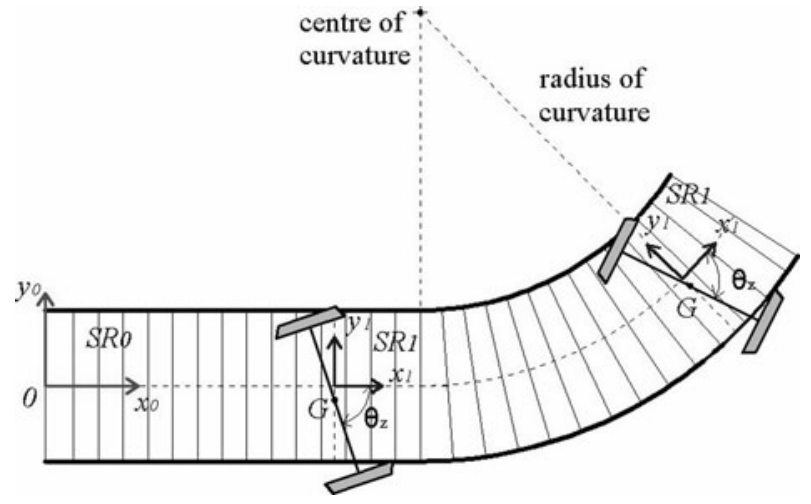

The fixed inertial reference system $S R_{0}$ has its origin on the rail longitudinal plane of symmetry and on the rolling plane, the axis $x_{o}$ is tangent to the railway path. The axis $z_{o}$ is vertical and directed upward.

The coordinate systems $S R_{G \text { front }}$ and $S R_{\text {back }}$ are framed with the front and back wheelsets, respectively. Their origins are coincident with their centers of masses. The axes $y_{G \text { front }}$ and $y_{G \text { back }}$ are coincident with the wheelsets axes, whereas the axes $z_{G \text { front }}$ and $z_{G \text { back }}$ are initially vertical and directed upwards.

The coordinate system $S R_{G \mathrm{fr}}$ is fixed with the bogie frame mass. Its origin is coincident with the bogie frame center of mass. The axis $x_{G \mathrm{fr}}$ is initially parallel to the rail direction, the axis $z_{G \text { fr }}$ is initially vertical and directed upwards.

The two reference systems attached to the wheelsets- $S R_{1 \text { front }}$ and $S R_{1 \text { back }}$ for the front and back one, respectively, have their origins belonging to the track longitudinal plane of symmetry and to the rolling plane. The origins of the two coordinate systems follow the path of the centers of mass of the wheelsets (see Fig. 3). Therefore, when the rail path is straight, the positions of the two frames have the same $x$ coordinates of the wheelset centers of mass.

The axes $x_{1 \text { front }}$ and $x_{1 \text { back }}$ are parallel to the rail direction. The axes $z_{1 \text { front }}$ and $z_{1 \text { back }}$ are orthogonal to the rolling plane and directed upward. Figure 1 locates all the coordinate systems introduced.

In order to obtain the transformation matrix between two generic coordinate systems, the following convention regarding the rotation angles has been adopted: given the two coordinate systems $\boldsymbol{S}_{a}$ and $\boldsymbol{S}_{b}, \boldsymbol{S}_{a}$ is assumed fixed while $\boldsymbol{S}_{b}$ is moving with the bogie.

Let us denote by $\alpha_{x}, \alpha_{y}, \alpha_{z}$ the Cardan angles which define the attitude of $\boldsymbol{S}_{b}$ with respect to $S_{a}$.

The rotation order with which $\boldsymbol{S}_{a}$ must rotate in order to superimpose himself on $\boldsymbol{S}_{b}$ is the following:

- I rotation about axis $z_{a}$ belonging to $S_{a}$. Under this rotation axes $x$ and $y$ move to $x^{\prime}$ and $y^{\prime}$, respectively.

- II rotation about axis $x^{\prime}$ belonging to the intermediate frame. Under this rotation axis $y^{\prime}$ moves to $y^{\prime \prime}$.

- III rotation about axis $y^{\prime \prime}$ coincident with $y_{b}$. 
In this manner, the matrix which transforms the vector components from $\boldsymbol{S}_{b}$ to $\boldsymbol{S}_{a}$ has the following expression:

$A_{\mathrm{rot}}^{S_{b} \rightarrow S_{a}}=\left[\begin{array}{ccc}\cos \alpha_{z} \cos \alpha_{x} & -\cos \alpha_{z} \cos \alpha_{y} \sin \alpha_{x}+\sin \alpha_{z} \sin \alpha_{y} & \cos \alpha_{z} \sin \alpha_{x} \sin \alpha_{y}+\cos \alpha_{y} \sin \alpha_{z} \\ \sin \alpha_{x} & \cos \alpha_{x} \cos \alpha_{y} & -\sin \alpha_{y} \cos \alpha_{x} \\ -\sin \alpha_{z} \cos \alpha_{x} & \sin \alpha_{z} \cos \alpha_{y} \sin \alpha_{x}+\sin \alpha_{y} \cos \alpha_{z} & -\sin \alpha_{x} \sin \alpha_{y} \sin \alpha_{z}+\cos \alpha_{y} \cos \alpha_{z}\end{array}\right]$.

Hence, the relation between the vector components in the two reference systems is:

$$
\vec{V}_{S_{a}}=A_{\mathrm{rot}}^{S_{b} \rightarrow S_{a}} \vec{V}_{S_{b}}
$$

where

$\vec{V}_{S_{a}}$ is the vector $\vec{V}$ expressed in $S_{a}$;

$\vec{V}_{S_{b}}$ is the vector $\vec{V}$ expressed in $\boldsymbol{S}_{b}$.

The analyses regard both straight and curved paths. In the first case, making reference to the motion of a single wheelset, the frame $S R_{1}$ translates with respect to $S R_{0}$ (see Fig. 1). When the track is curved, the motion of the frame $S R_{1}$ is characterized by a translational motion together with a rotational one, such that $x_{1}$ axis is tangent to the rail track centerline and $y_{1}$ axis points toward the center of curvature. In this case, the reading of the lookup tables is done with reference to the relative attitude of the frame $S R_{G}$ - fixed with the wheelset-with respect to the frame $S R_{1}$; then, the relative position and orientation of $S R_{G}$ with respect to $S R_{1}$ and all the data obtained from the lookup tables are transformed with respect to $S R_{0}$ by means of the transformations previously reported.

\section{The equations of motion of the bogie}

\subsection{The Newton-Euler equations}

The equations of dynamics are written only for the main three moving masses. The inertia of the suspension deformable elements is neglected.

The equations are expressed in the inertial coordinate system $S R_{0}$ and are given below:

$$
\vec{F}_{\mathrm{ext}}^{i}=m_{i} \vec{a}_{G}^{i} ; \quad \operatorname{ext}_{G}^{i}=\frac{d \vec{K}_{G}^{i}}{d t} ; \quad \vec{F}_{\mathrm{ext}}^{\mathrm{fr}}=m_{\mathrm{fr}} \vec{a}_{G}^{\mathrm{fr}} ; \quad \overrightarrow{e x t}_{G}^{\mathrm{fr}}=\frac{d \vec{K}_{G}^{\mathrm{fr}}}{d t} .
$$

Here subscript or superscript $i$ can assume the meaning of "front" or "back" when denoting the front or back wheelset, respectively;

$\vec{F}_{\text {ext }}^{i} \quad$ is the sum of all external forces acting on the $i$ th wheelset;

$\vec{a}_{G}^{i} \quad$ is the acceleration of the barycenter of the $i$ th wheelset;

$m_{i} \quad$ is the mass of the $i$ th wheelset;

${ }_{\text {ext }} \vec{M}_{G}^{i}$ is the resultant external moment about the barycenter acting on the $i$ th wheelset;

$\vec{K}_{G}^{i} \quad$ is the angular momentum about the barycenter of the $i$ th wheelset;

$\vec{F}_{\text {ext }}^{\text {fr }} \quad$ is the sum of all the external forces acting on the bogie frame;

$m_{\text {fr }}$ is the bogie frame mass;

$\vec{K}_{G}^{\mathrm{fr}} \quad$ is the angular momentum about the center of mass of the bogie frame;

${ }_{\text {ext }} \vec{M}_{G}^{\mathrm{fr}}$ is the resultant of all external forces moments about the center of mass and acting on the bogie frame. 


\subsection{The external forces and moments}

The external forces acting on the wheelsets and on the bogie frame are obtained from the following sums:

$$
\begin{aligned}
& \vec{F}_{\mathrm{ext}}^{i}=\vec{P}_{i}+\sum_{h=1}^{N_{l}} \overrightarrow{\mathrm{F}}_{i}^{h}+\sum_{k=1}^{N_{r}} \overrightarrow{\mathrm{Fr}}_{i}^{k}+\vec{F}_{i}^{\text {susp }}+\vec{F}_{i}^{o}, \\
& \vec{F}_{\mathrm{ext}}^{\mathrm{fr}}=\vec{P}_{\mathrm{fr}}+\vec{F}_{\mathrm{fr}}^{\text {susp }}+\vec{F}_{\mathrm{fr}}^{o},
\end{aligned}
$$

where

$\vec{P}_{i} \quad$ is the weight of the $i$ th wheelset;

${ }_{\text {cl }} \vec{F}_{i}^{h} \quad$ is the contact force acting on left wheel regarding the $h$ th contact point on the $i$ th wheelset;

${ }_{\text {cr }} \vec{F}_{i}^{k} \quad$ is the contact force acting on right wheel regarding the $k$ th contact point on the $i$ th wheelset;

$\vec{F}_{i}^{\text {susp }}$ is the sum of all forces acting on $i$ th wheelset and transmitted by the suspension system;

$\vec{F}_{i}^{o} \quad$ is the sum of all possible further external forces (pull force, load directly applied on the masses, etc.) acting on the $i$ th wheelset;

$\vec{P}_{\text {fr }} \quad$ is the weight of the bogie frame;

$\vec{F}_{\text {fr }}^{\text {susp }}$ is the sum of all forces acting on the bogie frame and transmitted through the suspension system;

$\vec{F}_{\text {fr }}^{o} \quad$ is the sum of the remaining external forces (e.g., pull force, load directly applied on the bogie frame, etc.) acting on bogie frame;

$N_{l}, N_{r}$ are the numbers of the contact points on the left and right wheels, respectively.

The resultant moments acting on the wheelsets and on the bogie frame are:

$$
\begin{aligned}
{ }_{\operatorname{ext}} \vec{M}_{G}^{i}= & \sum_{h=1}^{N_{l}}\left({ }_{\mathrm{cl}} G \vec{P}_{i}^{h} \times{ }_{\mathrm{cl}} \vec{F}_{i}^{h}+{ }_{i}^{\mathrm{cl}} \vec{M}_{\mathrm{spin}}^{h}\right)+\sum_{k=1}^{N_{r}}\left({ }_{\mathrm{cr}} G \vec{P}_{i}^{k} \times{ }_{\mathrm{cr}} \vec{F}_{i}^{k}+{ }_{i}^{\mathrm{cr}} \vec{M}_{\mathrm{spin}}^{k}\right) \\
& +{ }_{i} \vec{M}_{G}^{\mathrm{susp}}+{ }_{i} \vec{M}_{G}^{o}+{ }_{i} \vec{M}_{G}^{\mathrm{mot}} \\
\operatorname{ext} \vec{M}_{G}^{\mathrm{fr}}= & { }_{\mathrm{fr}} \vec{M}_{G}^{\mathrm{susp}}+{ }_{\mathrm{fr}} \vec{M}_{G}^{o},
\end{aligned}
$$

where

${ }_{\text {cl }} G \vec{P}_{i}^{h}$ is the vector joining the barycenter of the $i$ th wheelset with the $h$ th contact point on the left wheel of the $i$ th wheelset;

${ }_{\text {cr }} G \vec{P}_{i}^{k}$ is the vector joining the barycenter of the $i$ th wheelset with the $k$ th contact point on the right wheel of the $i$ th wheelset;

${ }_{i}^{\mathrm{cl}} \vec{M}_{\text {spin }}^{h}$ is the spin moment acting just on the $h$ th contact point on the left wheel of the $i$ th wheelset;

${ }_{i}^{\mathrm{cr}} \vec{M}_{\text {spin }}^{k}$ is the spin moment acting just on the $k$ th contact point on the right wheel of the $i$ th wheelset;

${ }_{i} \vec{M}_{G}^{\text {susp }}$ is the resultant moment about the center of mass of the forces transmitted by the suspension system to the $i$ th wheelset;

${ }_{i} \vec{M}_{G}^{o} \quad$ is the resultant moment about the center of mass of all possible further external forces acting on the $i$ th wheelset;

${ }_{i} \vec{M}_{G}^{\text {mot }}$ is the engine torque applied on the $i$ th wheelset; 


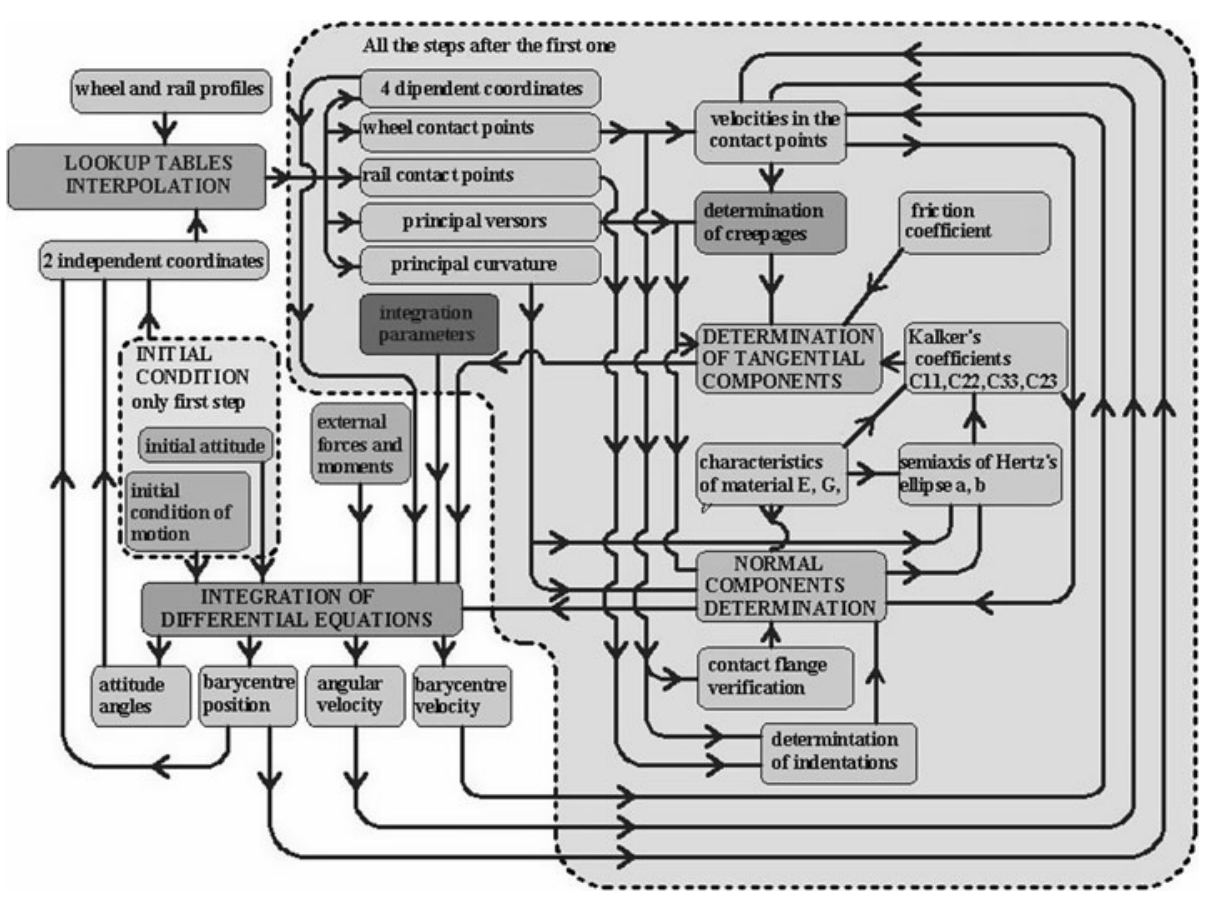

Fig. 4 Flow chart of the wheelset dynamic analysis

${ }_{\text {fr }} \vec{M}_{G}^{\text {susp }}$ is the resultant moment about the center of mass of the forces transmitted by the suspension system to the bogie frame;

${ }_{\mathrm{fr}} \vec{M}_{G}^{o} \quad$ is the resultant moment about the center of mass of the remaining external forces acting on the bogie frame.

Figure 4 shows the block-diagram used for the dynamic analysis of each wheelset. Therefore, in this diagram the wheelset is assumed as isolated from the main parts. The gray shaded area on the right side concerns the contact analysis. The left side reports the computational steps of the dynamic analysis.

\section{Contact points detection}

\subsection{The contact points}

With reference to the front wheelset [5], we consider the two volumes of intersection, $V_{\text {int }}^{r}$ and $V_{\text {int }}^{w}$, related to the rail and to the wheel, respectively. The two contact points are made coincident with the load center $P_{G w}$ and $P_{G r}$ of the grid nodes on the boundary of $V_{\text {int }}^{w}$ and $V_{\text {int }}^{r}$, respectively. Analytically, this results into:

$$
\overrightarrow{O P}_{G w}=\frac{\sum_{i=1}^{n_{w}} \overrightarrow{O P}_{i}^{w}}{n_{w}} ; \quad \overrightarrow{O P}_{G r}=\frac{\sum_{j=1}^{n_{r}} \overrightarrow{O P}_{j}^{r}}{n_{r}}
$$

where 
Fig. 5 Wheel/rail intersecting volume in the method of barycenter

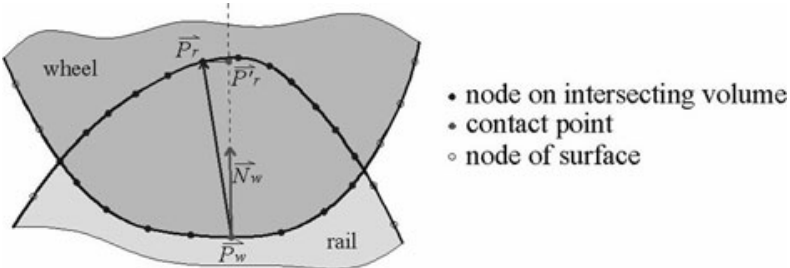

$\overrightarrow{O P}_{G w}$ and $\overrightarrow{O P}_{G r}$ denote the position vectors of the barycentres of the grid nodes on the boundaries of $V_{\text {int }}^{w}$ and $V_{\text {int }}^{r}$, respectively;

$\overrightarrow{O P}_{i}^{w}$ and $\overrightarrow{O P}_{j}^{r}$ denote the position vectors of generic grid nodes on the boundaries of $V_{\text {int }}^{w}$ and $V_{\text {int }}^{r}$, respectively;

$n_{w}$ and $n_{r}$ denote the nodes number of the intersection volumes of the wheel and the rail, respectively.

The maximum indentation $C_{\max }$ is calculated by means of the formula:

$$
C_{\max }=\left(\overrightarrow{O P}_{G r}-\overrightarrow{O P}_{G w}\right) \cdot \vec{N}_{w} .
$$

Since $P_{G w}$ does not belong to the wheel surface, the normal unit vector $\vec{N}_{w}$ coincides with the normal to the wheel surface passing through $P_{G w}$, as shown in Fig. 5.

\subsection{The compilation of lookup tables}

The knowledge of the contact features, such as contact point positions, the normal unit vectors and the principal curvatures of interactive surfaces, is very important in the dynamics analysis. These calculations introduce a computationally intensive workload on the computer cpu. In an effort to reduce such workload, lookup tables have been compiled before running the simulation. These are matrices determined, once and for all, for a given wheel and rail profiles, the matrices are continuously queried during the simulation time steps.

The unconstrained wheelset has six degrees of freedom. Therefore, its spatial location is specified by the three coordinates $x_{G}, y_{G}, z_{G}$ of the barycenter and by the three Cardan angles $\alpha_{x}, \alpha_{y}, \alpha_{z}$ specifying the attitude of frame $S R_{G}$ (fixed with the wheelset) with respect to $S R_{0}$. When the wheelset is in contact with the rails, the degrees of freedom reduce to two. Hence, during the compilation of the lookup table, two independent variables are specified, namely the transverse displacement of wheelset barycenter, $y_{G}$, and the yaw angle $\alpha_{z}$. The remaining dependent coordinates, i.e., $x_{G}, z_{G}, \alpha_{x}, \alpha_{y}$, are obtained by imposing the equilibrium conditions to the wheelset under the action of external forces. The values of the two independent coordinates are both uniformly spaced within intervals extended to the typical range of values experienced during the contact between rails and wheels. In particular, the interval extremes are:

$$
-9 \mathrm{~mm} \leq y_{G} \leq 9 \mathrm{~mm} ; \quad-0.12 \mathrm{rad} \leq \alpha_{z} \leq 0.12 \mathrm{rad} .
$$

Having subdivided the intervals into 36 parts, the values of dependent coordinates, together with all information regarding contact, are here stored in $37 \times 37$ matrices, with different values for right and left wheels. The contact features stored in the lookup tables are: 
Fig. 6 Coordinates $x$ of the contact point on the left (a) and right wheel (b)

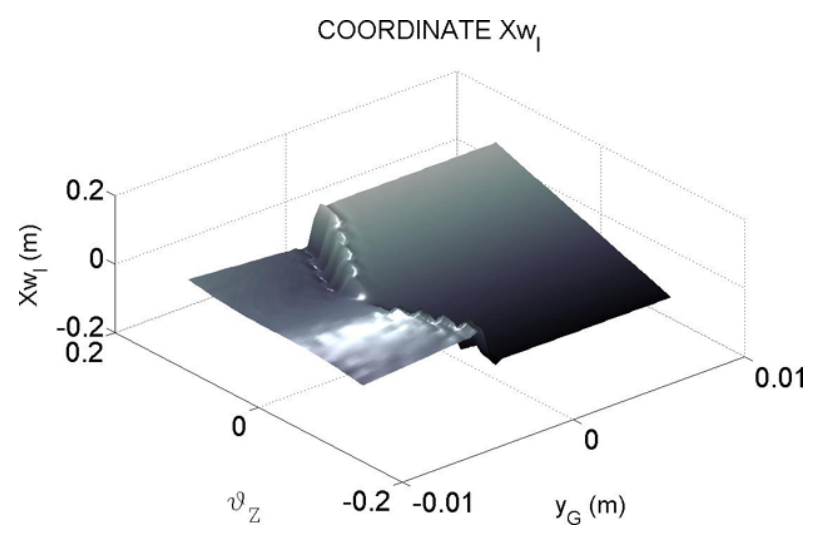

(a)

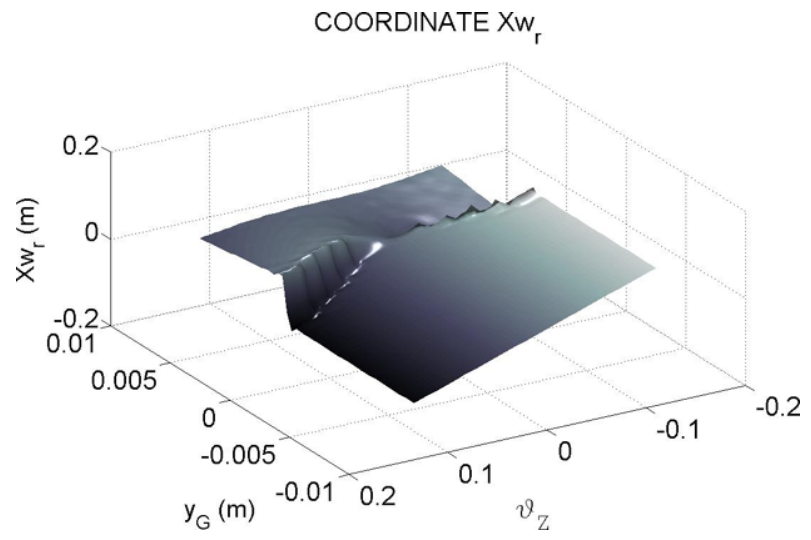

(b)

- Four dependent coordinates;

- Coordinates of the contact point on the wheel;

- Coordinates of the contact point on the rail;

- Components of the longitudinal, transverse and normal unit vectors on the wheel in correspondence to the contact point;

- Components of the longitudinal, transverse and normal unit vectors on the rail in correspondence to the contact point;

- Principal curvatures and directions on the wheel surface in the contact point;

- Principal curvatures and directions on the rail surface in the contact point.

As an example, Figs. 6(a) and 6(b) show the $x$ coordinate of the contact points on the left and right wheels, respectively, expressed in the $S R_{1 \text { Front }}$ reference system.

From the figures, we can highlight that the values of the $x$ coordinates have a rapid variation when the contact goes from the tread to the flange of the wheel. We also observe that the value referred to the two wheels have characteristics of symmetry and anti-symmetry with respect to the two independent coordinates $y_{G}$ and $\alpha_{z}$. 


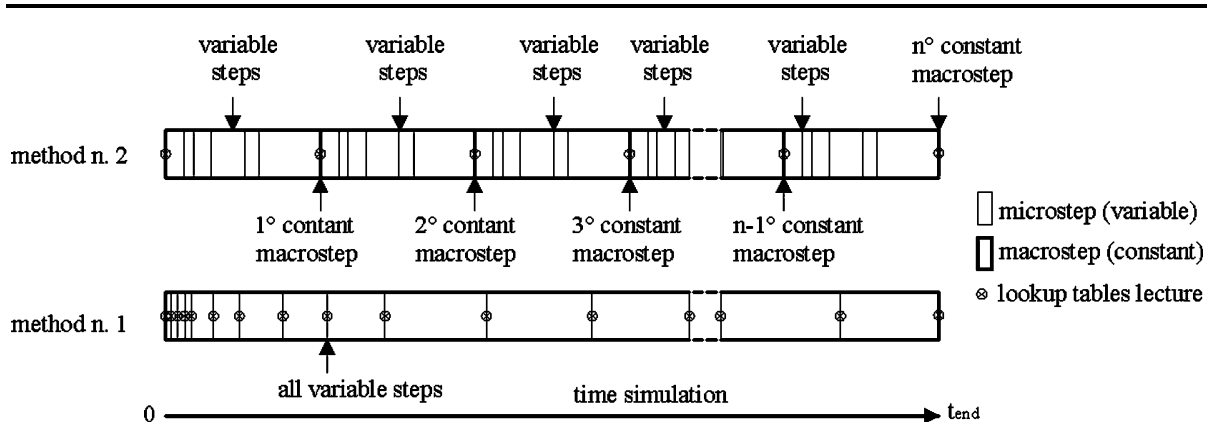

Fig. 7 The two query methods of the lookup tables

\subsection{Use of lookup tables}

Given the generic values $y_{G}$ and $\alpha_{z}$, the corresponding values of $x_{G}, z_{G}, \alpha_{x}, \alpha_{y}$ are computed by means of linear interpolation of the values stored in the lookup tables.

The query of the values can be done through two different strategies:

- Method 1: at each integration step;

- Method 2: at previously established steps (macrosteps).

The use of tabulated variables has already been successfully adopted in the field of wheelrail contact analysis [4]. This approach is not recommended for studying the extreme dynamic simulations such as accident events or derailments. In this investigation, the method of calculating the values stored in the lookup table is different from the one used in [4].

In the first method, the query to the lookup table occurs every time the Matlab procedure, namely ODE23t, evaluates the differential equations. The ODE solver herein adopted is suitable for moderately stiff problems without numerical damping. Alternatively, with the second method, a constant macrostep is introduced. Within the macrostep, the dependent values are not updated. When switching from one macrostep to the next one, the coordinates at the end of a macrostep are considered as initial conditions for the next time interval. The two different strategies are sketched in Fig. 7.

With the aim of finding a trade-off method for sparing on cpu time and enhancing data accuracy, the results of the two approaches have been compared with those obtained with a commercial multibody software. For the second method of query, we have adopted three different macrosteps, i.e., $10^{-4}, 4 \cdot 10^{-4}, 4.2 \cdot 10^{-4}$ s.

Figure 8(a) shows the transverse displacement of the wheelset moving with a longitudinal speed equal to $12 \mathrm{~m} / \mathrm{s}$, initially perturbed through a transverse velocity of $0.1 \mathrm{~m} / \mathrm{s}$. Figure 8(b) shows the absolute value of the differences obtained by comparing the two method results and those given by commercial multibody software.

The absolute value of the relative difference at time $t$ is calculated as follows:

$$
e r r_{\text {rel }}^{\mathrm{abs}}(t)=\left\|\frac{f(t)-g(t)}{g(t)}\right\|,
$$

while the mean of $e r r_{\text {rel }}^{\mathrm{abs}}$ on an interval $\left(t_{1}-t_{0}\right)$ is

$$
\text { mean }_{\mathrm{rel}}=\frac{\int_{t_{0}}^{t_{1}} e r r_{\mathrm{rel}}^{\mathrm{abs}}(t)}{\left(t_{1}-t_{0}\right)} .
$$




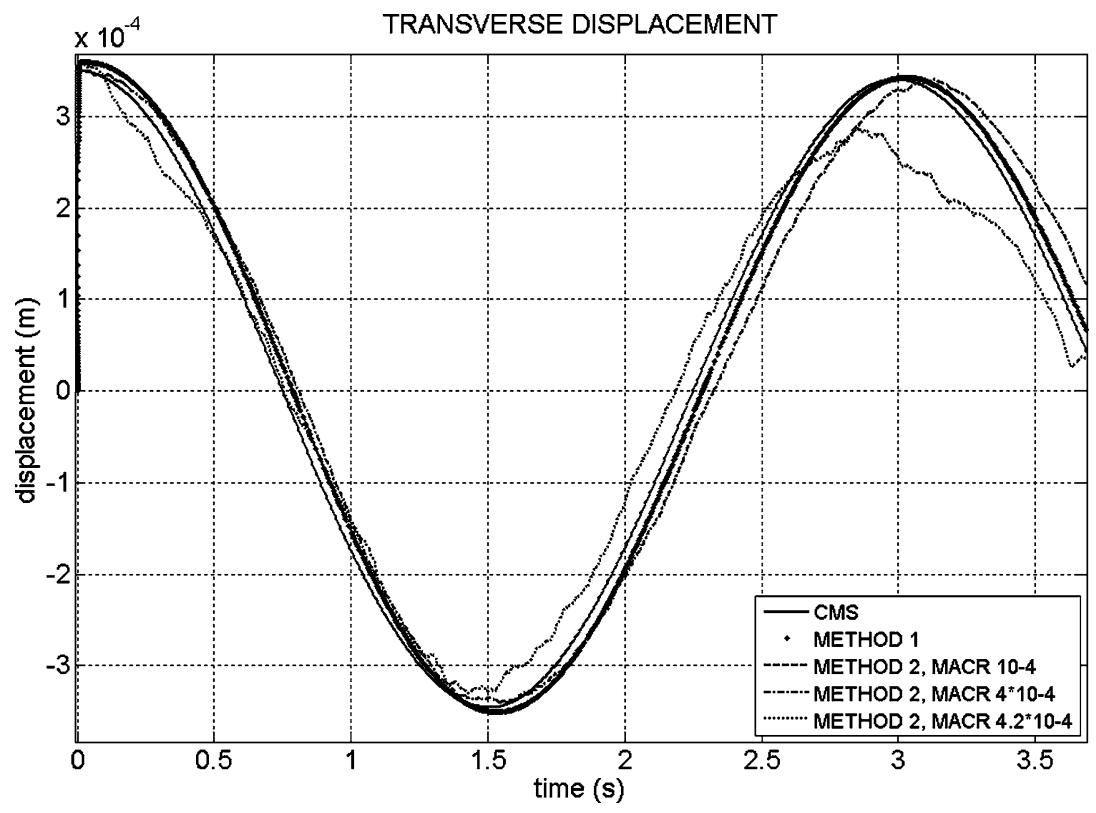

(a)

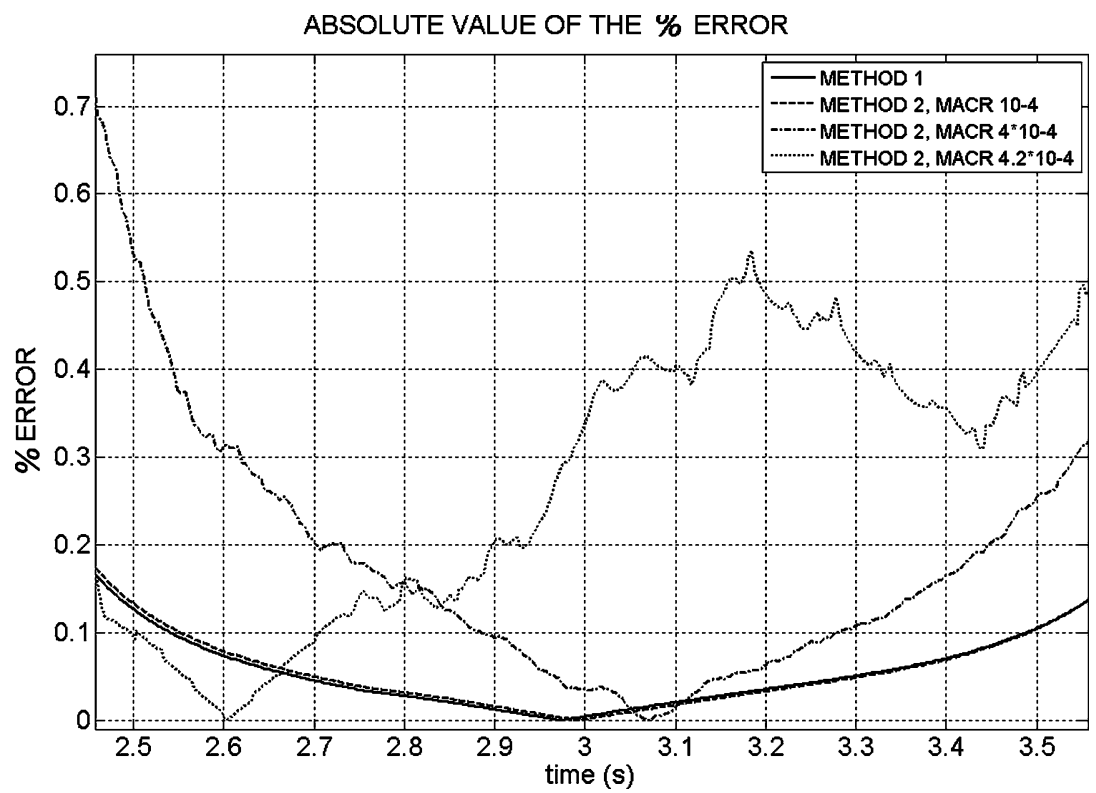

(b)

Fig. 8 The wheelset barycenter motion calculated by means of the two query methods compared with the commercial multibody software (CMS) results 
Table 4 Cpu time for $10 \mathrm{~s}$ simulation and mean relative error referred to the commercial multibody software or obtained by means of the two methods with three macrostep values

\begin{tabular}{llll}
\hline Method & & Cpu time (s) & Mean relative error \\
\hline Method 1 & & 346 & 0.53 \\
\hline Method 2 & Macrostep (s) & & \\
& $1 \cdot 10^{-4}$ & 8415 & 4.85 \\
& $4 \cdot 10^{-4}$ & 1122 & 1.71 \\
& $4.2 \cdot 10^{-4}$ & 1037 & 0.84 \\
\hline
\end{tabular}

In (8), $f(t)$ and $g(t)$ are the time functions to be compared. Table 4 shows the results of the two methods for different macrostep length. It is evident that the first method is the most accurate and (this is unexpected at first glance) rapid. For this reason, we have adopted it in all simulations. As a matter of fact, even if the increasing number of queries should require a longer cpu-time, the abrupt change of contact condition encountered in the second method implies a significant reduction in the time step (more iterations are needed for the same analysis) that masks the benefits of lighter queries.

\subsection{The interpolation of the lookup tables}

In order to optimize the interpolation of the values stored in the lookup tables, a new function called my_interp2.m has been developed in replacement of the two functions interp2.m and griddata.m from the standard Matlab library.

The interpolation is performed in two steps:

- Query of the matrices stored in the lookup tables;

- Linear interpolation of a reduced set of the retrieved values.

These $37 \times 37$ matrices are initially saved on the mass storage as a unique layered matrix. Herein we refer for generality to a generic $r \times m \times p$ matrix, where $r$ is the number of rows, $m$ the number of columns and $p$ the number of layers.

Let us denote by $x$ and $y$ the two coordinate axes related to the column and row directions, respectively. The values of $x_{i}$ and $y_{i}$, both monotonically varying, satisfy the following inequalities:

$$
\begin{aligned}
& x_{\min } \leq x_{i} \leq x_{\max }, \\
& y_{\min } \leq y_{j} \leq y_{\max },
\end{aligned}
$$

where

$x_{i}$ and $\mathrm{y}_{j}$ are the values of $x$ and $y$ corresponding to the $i$ th column and $j$ th row;

$x_{\min }$ and $x_{\max }$ are the minimum and maximum column values;

$y_{\min }$ and $y_{\max }$ are the minimum and maximum row values;

$i \in[1, m]$ and $j \in[1, r]$ are integer subscripts.

In order to reduce cpu-time, a block matrix [A] of dimensions $2 \times 2 \times p$ is extracted from the whole matrix containing the lookup table. Let us denote by $c_{x}$ and $c_{y}$ the generic values of the independent coordinates. At the row and column boundaries of the block matrix, there are the stored independent coordinates adjacent to $c_{x}$ and $c_{y}$ such that (see Fig. 9)

$$
\begin{aligned}
& x_{n} \leq c_{x} \leq x_{n+1}, \\
& y_{h} \leq c_{y} \leq y_{h+1} .
\end{aligned}
$$



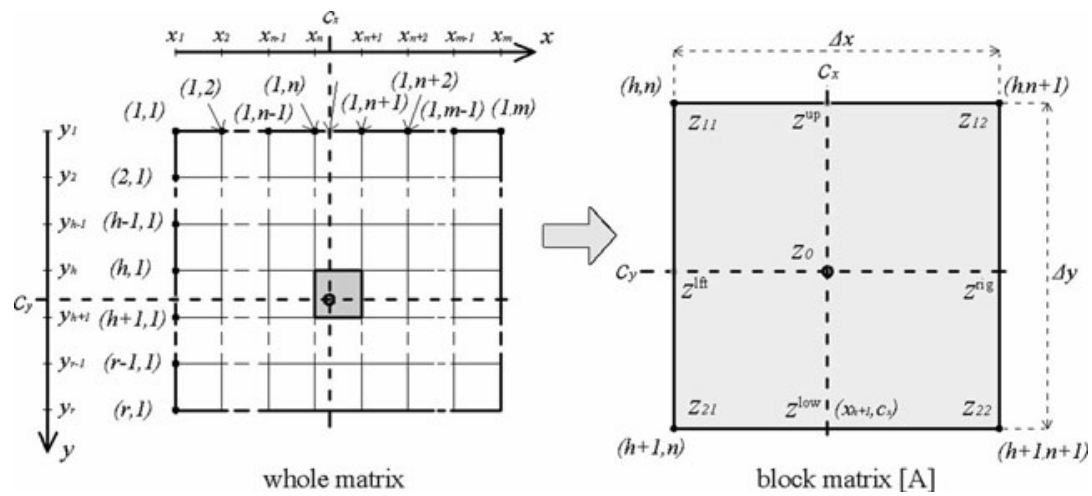

Fig. 9 Diagram of the interpolation method used for the lookup table

Given [A], the linear interpolation is applied. The following procedure refers only to a particular $k$-value $(k=1,2, \ldots, p)$ of the stored matrix.

Let us denote by:

- $z(y, x)$ the value of the matrix element corresponding to the $y$ and $x$ values and related to the directions of the row and the column;

$-z_{r s}=A(r, s, k)$ the value of the block matrix [A] element corresponding to the $r$ th row and $s$ th column.

Given the matrix of the whole lookup table and the values $c_{x}$ and $c_{y}$, the linearly interpolated value $z_{0}=z\left(c_{y}, c_{x}\right)$ is computed through the following steps:

1. Form the elements of the block matrix

$$
\begin{aligned}
& z_{11}=A(h, n, k) ; \quad z_{12}=A(h, n+1, k) ; \\
& z_{21}=A(h+1, n, k) ; \quad z_{22}=A(h+1, n+1, k) ;
\end{aligned}
$$

2. Let $\Delta_{x}=x_{i+1}-x_{i}$ be the constant column spacing and $\Delta_{y}=y_{j+1}-y_{j}$ the constant row spacing.

3. Evaluate $z^{\text {up }}=z\left(y_{h}, c_{x}\right)$ and $z^{\text {low }}=z\left(y_{h+1}, c_{x}\right)$ corresponding to the value $c_{x}$ by means of the following expressions:

$$
z^{\mathrm{up}}=z_{11}+\left(c_{x}-x_{n}\right) \frac{z_{12}-z_{11}}{\Delta_{x}} ; \quad z^{\text {low }}=z_{21}+\left(c_{x}-x_{n}\right) \frac{z_{22}-z_{21}}{\Delta_{x}} ;
$$

4. Evaluate the column $z_{0}$ value, namely $z_{0}^{x}$, by means of the following expression:

$$
z_{0}^{x}=z^{\mathrm{up}}+\left(c_{y}-y_{h}\right) \frac{z^{\mathrm{low}}-z^{\mathrm{up}}}{\Delta_{y}}
$$

5. Evaluate $z^{\mathrm{lft}}=z\left(c_{y}, x_{n}\right)$ and $z^{\text {rig }}=z\left(c_{y}, x_{n+1}\right)$ corresponding to the value $c_{y}$ by means of the following expressions:

$$
z^{\mathrm{lft}}=z_{11}+\left(c_{y}-y_{h}\right) \frac{z_{21}-z_{11}}{\Delta_{y}} ; \quad z^{\mathrm{rig}}=z_{12}+\left(c_{y}-y_{h}\right) \frac{z_{22}-z_{12}}{\Delta_{y}} ;
$$


6. Evaluate the row $z_{0}$, namely $z_{0}^{y}$, by means of the following expression:

$$
z_{0}^{y}=z^{\mathrm{lft}}+\left(c_{x}-x_{n}\right) \frac{z^{\mathrm{rig}}-z^{\mathrm{lft}}}{\Delta_{x}} ;
$$

7. Finally, the value of $z_{0}$ is given by:

$$
z_{0}=\frac{\left(z_{0}^{x}+z_{0}^{y}\right)}{2}
$$

Figure 9 shows the diagram of the whole and the partitioned matrix used for interpolation purposes.

In order to test the speedup obtained by adopting the new function, the cpu time requested for the interpolation of the two standard Matlab functions interp2 $\mathrm{m}$ and griddata.m and the new function my_interp $2 . \mathrm{m}$ is compared. The superiority of the proposed approach is demonstrated by the fact that using a $2 \times 2$ matrix the new function is almost 5.3 and 4.75 faster than interp $2 . \mathrm{m}$ and griddata.m, respectively.

\section{The contact forces}

\subsection{Classification of the contact forces}

The contact force $\vec{F}_{c}$ between the wheel and rail are split into normal and tangent components. The normal component $\vec{N}_{c}$ has the direction of the normal unit vector at the contact point. The current normal components are obtained adding spring $\vec{N}_{\text {el }}$ and damper $\vec{N}_{\text {visc }}$ forces. The tangent component lies in the tangent plane and is formed by two contributions: $\vec{F}_{\text {long }}$ directed along the longitudinal direction, and $\vec{F}_{\text {trans }}$ parallel to the transverse unit vector. The tangent component is estimated by means of Kalker linear theory [6].

In conclusion, the following relationships related to contact can be established, in addition to the spin moment expression:

$$
\vec{F}_{c}=\vec{N}_{c}+\vec{F}_{\text {tang }} ; \quad \vec{N}_{c}=\vec{N}_{\text {visc }}+\vec{N}_{\text {el }} ; \quad \vec{F}_{\text {tang }}=\vec{F}_{\text {long }}+\vec{F}_{\text {trans }} .
$$

7.2 Computation of the normal component $\vec{N}_{c}$

\subsubsection{The elastic contribution}

The elastic contribution $\vec{N}_{\text {el }}$ is expressed in the form

$$
\vec{N}_{\mathrm{el}}=k_{\mathrm{el}} C \vec{n},
$$

where

$\vec{n}$ is the normal unit vector to the surface in the contact point;

$C$ is the indentation between the wheel and rail;

$k_{\mathrm{el}}$ is the elastic contact stiffnesses.

The value of $k_{\mathrm{el}}$ depends on the location of the contact area:

- Contact in the wheel tread;

- Contact in the wheel flange. 
${ }^{1}$ NODAL SOLUTION

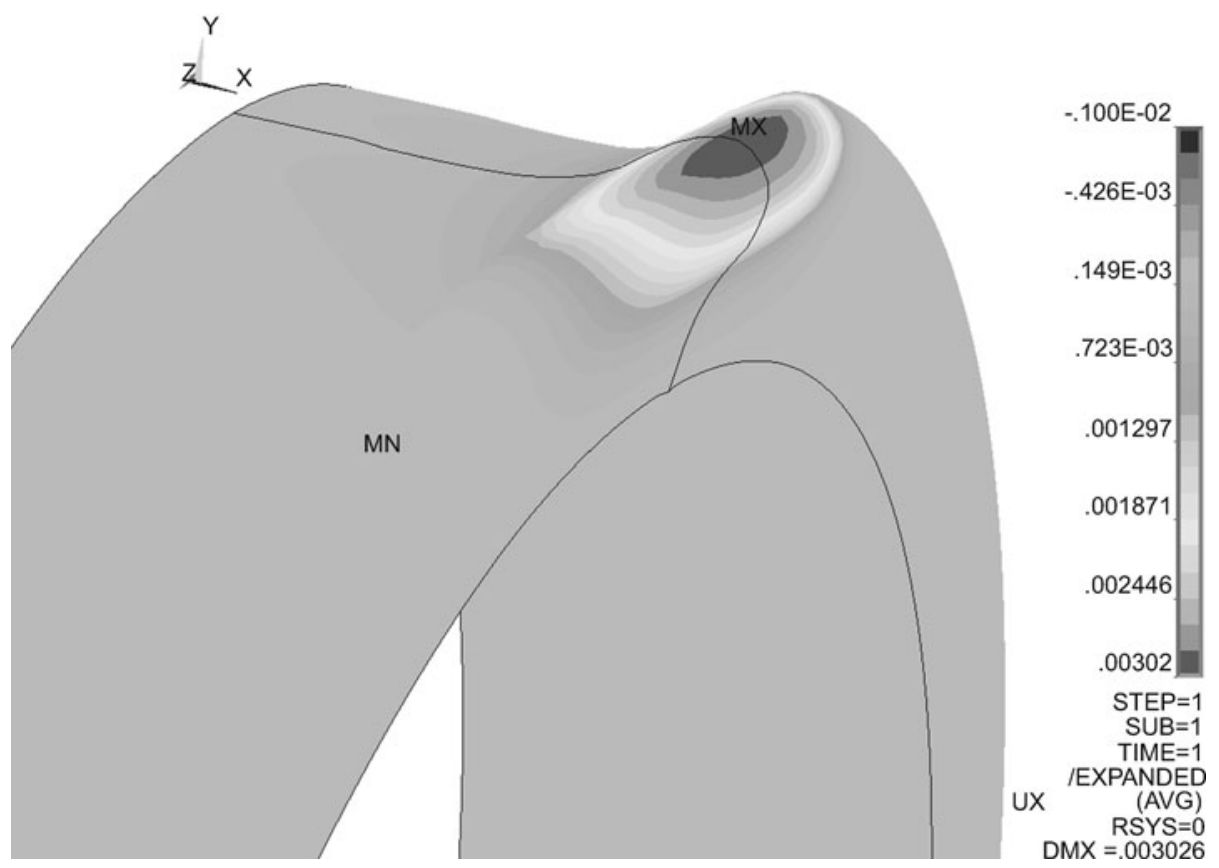

Fig. 10 Displacement field of the wheel when in top contact with the rail

Fig. 11 Contributions to the total displacement of the flange

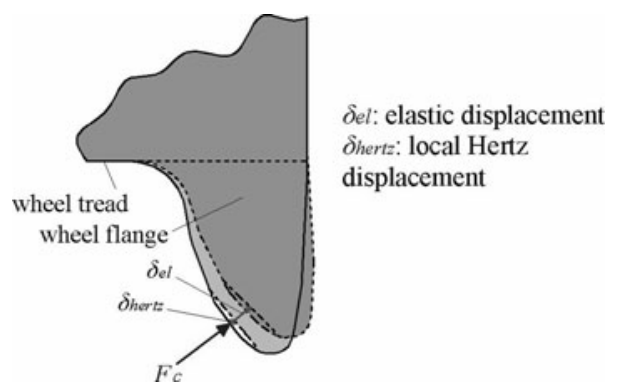

In the case of tread contact, we take into account only Hertz solution for the elastic stiffness evaluation. This depends only on material and local surface curvatures.

When the contact occurs in the flange, two terms are considered. The first one is due to Hertz deformation, the second one belongs to the deformability of an axial symmetric body (the wheel) loaded through the contact on a cylindrical body (the rail). It is not easy to determine this deformability in an analytical way. An appropriate finite element solution was performed to get an equivalent cantilever beam (a beam clamped at one end and loaded on the other free end) that allows accounting of the flange stiffness when loaded at different heights. In Fig. 10, the finite element analysis results, in terms of displacement field, are shown. Using these results, a suitable value for the width $b$ of an equivalent cantilever beam was found.

Figure 11 isolates the two contributions to the overall displacement when a force is applied close to the tip of the flange. 
According to the tuned cantilever beam model, the body stiffness $k_{\mathrm{el}}$ varies linearly along the flange.

The maximum value for the overall stiffness $k_{\max }$ is on the tread, whereas the minimum value $k_{\min }$ is at the flange tip. In our analysis, $k_{\max }$ is calculated by accounting the Hertz deformation only. Here the nonlinearity of the function load-displacement is not considered because a given (unit) value of the indentation is assumed, whatever is the point of contact. Stiffness $k_{\min }$ includes also the deformation of the flange modeled as cantilever beam.

Let us denote by:

$G$ the tangent module of elasticity;

$v$ the Poisson coefficient;

$C_{\text {long }}^{w}$ and $C_{\text {long }}^{r}$ the longitudinal curvatures at contact point of the wheel and the rail;

$C_{\text {trans }}^{w}$ and $C_{\text {trans }}^{r}$ the transverse curvatures at contact point of the wheel and the rail;

$$
\begin{aligned}
& D_{\text {trans }}=\frac{1}{2}\left(C_{\text {trans }}^{w}+C_{\text {trans }}^{r}\right) ; \\
& D_{\text {long }}=\frac{1}{2}\left(C_{\text {long }}^{w}+C_{\text {long }}^{r}\right) ; \\
& D_{\max }=\max \left(D_{\text {long }}, D_{\text {trans }}\right) ; \\
& D_{\text {min }}=\min \left(D_{\text {long }}, D_{\text {trans }}\right) .
\end{aligned}
$$

Hertz stiffness is computed by means of the formula [9]:

$$
K_{\text {hertz }}=\frac{2 \pi G}{3(1-v)} \frac{1}{\sqrt{D_{\max }+D_{\min }}} \frac{\sqrt{E(g)}}{g W(g)^{\frac{3}{2}}},
$$

where $E(g)$ and $W(g)$ are elliptic integrals defined as follows:

$$
\begin{aligned}
& W(g)=\int_{0}^{\pi / 2}\left[1-\left(1-g^{2}\right) \sin ^{2} \psi\right]^{-1 / 2} d \psi, \\
& E(g)=\int_{0}^{\pi / 2}\left[1-\left(1-g^{2}\right) \sin ^{2} \psi\right]^{1 / 2} d \psi,
\end{aligned}
$$

$g=\min \left(\frac{a}{b}, \frac{b}{a}\right)$ with $a$ and $b$ being contact ellipse semi-axes lengths of the approximated elliptic contact area according to the Hertz model.

Replacing the values of the curvatures at the base and tip of flange, one obtains:

$$
k_{\text {hertz }}^{\text {bf }}=7.87 \cdot 10^{10} \frac{\mathrm{N}}{\mathrm{m}} ; \quad k_{\text {hertz }}^{\mathrm{tf}}=7.09 \cdot 10^{9} \frac{\mathrm{N}}{\mathrm{m}} ;
$$

where $k_{\text {hertz }}^{\text {bf }}$ and $k_{\text {hertz }}^{\text {tf }}$ are the stiffnesses due to Hertz deformation at the base of the flange and at the tip.

The second contribution $k_{\mathrm{el}}^{\mathrm{tf}}$ is obtained by considering the wheel flange as a simple cantilever beam with the following dimensions (see Fig. 12 for nomenclature):

$$
\begin{aligned}
& b=0.032 \mathrm{~m} ; \quad h=0.045 \mathrm{~m} ; \quad l=0.0275 \mathrm{~m} ; \\
& k_{\mathrm{el}}^{\mathrm{tf}}=\frac{b h^{3} E}{4 l^{3}}=7.36 \cdot 10^{9} \frac{\mathrm{N}}{\mathrm{m}} .
\end{aligned}
$$


Fig. 12 Model of the flange as a cantilever beam

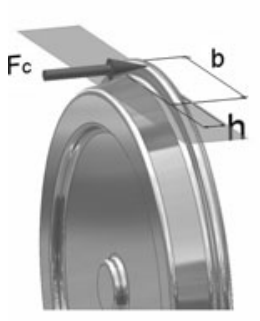

b)

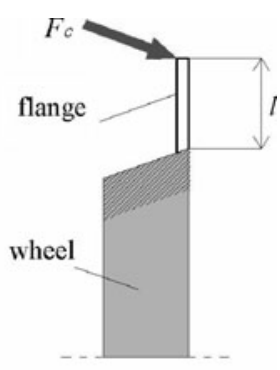

a)

Fig. 13 Variation of contact stiffness $k_{c}$ according to the position of the contact point $P_{c}$

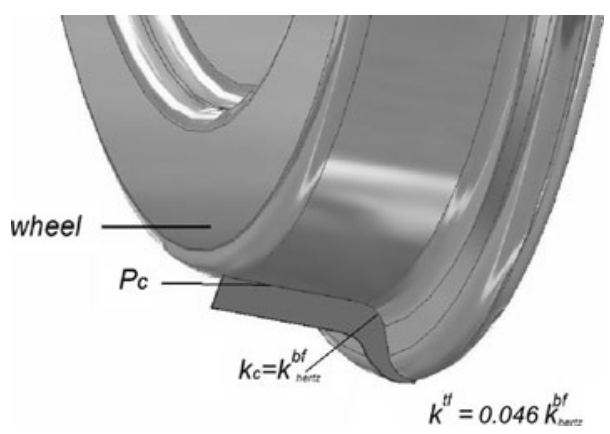

The overall stiffness at the flange tip is

$$
k^{\mathrm{tf}}=\frac{k_{\mathrm{hertz}}^{\mathrm{tf}} k_{\mathrm{el}}^{\mathrm{tf}}}{k_{\text {hertzx }}^{\mathrm{ff}}+k_{\mathrm{el}}^{\mathrm{tf}}}=3.61 \cdot 10^{9} \frac{\mathrm{N}}{\mathrm{m}} .
$$

It should be observed that the considerable ratio between the stiffnesses resulting at the tip and the base of the flange is

$$
\chi=\frac{k^{\mathrm{tf}}}{k_{\text {hertz }}^{\mathrm{bf}}}=0.046 .
$$

The magnitude of this ratio, though significant, seems neglected in the models available in the current technical literature.

The intermediate stiffnesses values within the base and the tip of the flange are calculated by means of a linear interpolation (see Fig. 13).

\subsubsection{The damping contribution}

The contribution to contact forces due to damping is proportional to the interpenetrating velocity $v_{\text {pen }}$ of the wheel in the rail. One can use the following expressions:

$$
\vec{N}_{\text {visc }}= \begin{cases}c_{v} v_{\text {pen }} \vec{n}, & v_{\text {pen }}>0, \\ 0, & v_{\text {pen }} \leq 0\end{cases}
$$

where

$$
\begin{aligned}
& v_{\text {pen }}=\vec{V}_{P} \cdot \vec{n}, \\
& \vec{V}_{P}=\vec{V}_{G}+\vec{\omega} \times G \vec{P} .
\end{aligned}
$$


The previous symbols have the following meaning:

$c_{v} \quad$ is the damping coefficient;

$\vec{V}_{P}$ is the velocity of the wheel in correspondence of the contact point;

$\vec{n} \quad$ is the normal unit vector in the contact point;

$\vec{V}_{G}$ is the velocity of the barycenter of the wheelset;

$\vec{\varpi} \quad$ is the angular velocity of the wheelset;

$G \vec{P}$ is the vector joining the barycenter of the wheelset with the contact point $P$.

The damping coefficient $c_{v}$ depends on the material of the solids in contact, here a standard steel-steel contact is assumed.

\subsection{Wheel-rail tangent component analysis}

The tangent component of the contact force is determined by means of the Kalker linear theory [6]. This is based on a linear relationship between the creepage and the tangent force. The contributions along the longitudinal, transverse and normal direction are given by:

$$
\begin{aligned}
& \vec{F}_{\text {long }}=-\left(c^{2} G C_{11} \zeta\right) \vec{n}_{\text {long }}, \\
& \vec{F}_{\text {trans }}=-\left(c^{2} G C_{22} \eta+c^{3} G C_{23} \varphi\right) \vec{n}_{\text {trans }}, \\
& \vec{M}_{\text {spin }}=-\left(c^{3} G C_{32} \eta+c^{4} G C_{33} \varphi\right) \vec{n}_{\text {norm }},
\end{aligned}
$$

where

$$
c=\sqrt{a b} ; \quad G=\left[\frac{1}{2}\left(\frac{1}{G_{1}}+\frac{1}{G_{2}}\right)\right]^{-1} .
$$

The symbols have the following meaning:

$G_{1}$ and $G_{2}$ are the wheel and the rail tangent elasticity modules, respectively;

$\zeta, \eta, \varphi$ are the longitudinal, transverse and spin creepages of the wheel respect to the rail at the contact point;

$C_{11}, C_{22}, C_{33}, C_{23}, C_{32}$ are the Kalker coefficients functions of the ratio $a / b$ and Poisson module;

$\vec{n}_{\text {long }}, \vec{n}_{\text {trans }}, \vec{n}_{\text {norm }}$ are the longitudinal, transverse and normal unit vectors at contact points.

The intensity of the tangent component, in the case of unlimited linear behavior, is given by

$$
F_{\text {tan }}=\left\|\vec{F}_{\text {tan }}\right\|=\sqrt{F_{\text {long }}^{2}+F_{\text {trans }}^{2}} .
$$

However, the magnitude of the tangent component should not exceed the pure slip value that depends on the adhesion coefficient $\mu$ (in our analysis, it is assumed that $\mu=0.2$ ):

$$
F_{\tan }^{\lim }=\mu N
$$

where $N$ is the normal force component.

The tangent component given by the linear model can be used for the nonlinear model by introducing the expressions:

$$
\vec{F}_{\text {long }}^{n l}=\varepsilon \vec{F}_{\text {long }} ; \quad \bar{F}_{\text {trans }}^{n l}=\varepsilon \vec{F}_{\text {trans }},
$$


Fig. 14 Velocity of the wheelset in the contact point and principal versors

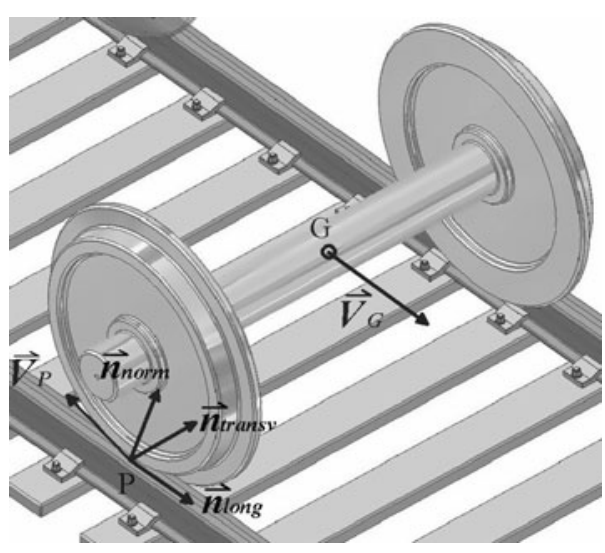

with the saturation coefficient $\varepsilon$ computed according to the following equations:

$$
\varepsilon= \begin{cases}\frac{\mu N}{F_{\tan }}\left[\left(\frac{F_{\tan }}{\mu N}\right)-\frac{1}{3}\left(\frac{F_{\tan }}{\mu N}\right)^{2}+\frac{1}{27}\left(\frac{F_{\tan }}{\mu N}\right)^{3}\right], & F_{\tan } \leq 3 \mu \mathrm{N}, \\ \frac{\mu N}{F_{\tan }}, & F_{\tan }>3 \mu \mathrm{N} .\end{cases}
$$

\section{The creepages}

In order to define the creepages between wheel and rail, we consider the wheel-rail relative velocity $\vec{V}_{P}$ at contact points. The creepages are classified in three different categories according to the directions interested:

- Longitudinal creepage $\zeta=\frac{\vec{V}_{P} \cdot \vec{n}_{\text {long }}}{\left\|V_{G}\right\|}$;

- Transverse creepage $\quad \eta=\frac{\vec{V}_{P} \cdot \vec{n}_{\text {trans }}}{\left\|V_{G}\right\|}$;

- Spin creepage $\quad \varphi=\frac{\vec{V}_{P} \cdot \vec{n}_{\text {norm }}}{\left\|V_{G}\right\|}$

where

$\vec{n}_{\text {long }}, \vec{n}_{\text {trans }}, \vec{n}_{\text {norm }}$ are, respectively, the longitudinal, transverse and normal unit vectors; $\left\|\vec{V}_{G}\right\|$ is the Euclidean norm of the barycenter velocity of the wheelset.

In Fig. 14, the wheelset velocities at barycenter $G$, at contact point $P$ and the principal contact unit vectors are represented.

\section{Forces generated by the suspension system}

The suspension is modeled as a linear spring-damper element with constant parameters. Therefore, the forces due to elastic and damping effects are expressed by the following formulas

$$
\vec{F}_{\mathrm{el}}^{\text {susp }}=\left(l_{o}-A B\right) k_{\mathrm{el}} \vec{n}_{\mathrm{AB}} ; \quad \vec{F}_{v}^{\text {susp }}=c_{v}\left[\left(\vec{V}_{\mathrm{A}}-\vec{V}_{\mathrm{B}}\right) \cdot \vec{n}_{\mathrm{AB}}\right] \vec{n}_{\mathrm{AB}}
$$

where the symbols have the following meaning: 
$\vec{F}_{\text {el }}^{\text {susp }}$ is the elastic contribution to the suspension force;

$\vec{F}_{v}^{\text {susp }}$ is the viscous contribution to the suspension force;

$\mathrm{AB}$ is the length of the segment AB;

$k_{\mathrm{el}} \quad$ is the spring stiffness of the suspension;

$c_{v} \quad$ is the damping coefficient of the suspension;

$\vec{n}_{\mathrm{AB}}$ is the unit vector along the line through attachment points $\mathrm{A}$ and $\mathrm{B}$, oriented from $\mathrm{A}$ to $\mathrm{B}$;

$\vec{V}_{\mathrm{A}} \quad$ is the velocity of point A;

$\vec{V}_{\mathrm{B}}$ is the velocity of point $\mathrm{B}$;

$l_{o} \quad$ is the length of the unloaded elastic element.

\section{The differential equations}

The dynamic equilibrium conditions (3) form a system of 18 second order differential equations. The unknowns are the following coordinates of the moving bodies:

$x_{G}^{j}(t), y_{G}^{j}(t), z_{G}^{j}(t)$ coordinates of the barycenter of the $j$ th body with respect to $S R_{0}$; $\vartheta_{x}^{j}(t), \vartheta_{y}^{j}(t), \vartheta_{z}^{j}(t)$ attitude angles of the frame $S R_{G j}$, connected to the $j$ th body with respect to $S R_{0}$.

The subscript $j$ is equal to "front", "back" or " $f r$ " when the variable pertains to front and back wheelset, bogie frame, respectively.

The accelerations of the barycentres are given by the following relations:

$$
\vec{a}_{G}^{j}=\frac{d^{2} x_{G}^{j}}{d t^{2}} \vec{i}_{0}+\frac{d^{2} y_{G}^{j}}{d t^{2}} \vec{j}_{0}+\frac{d^{2} z_{G}^{j}}{d t^{2}} \vec{k}_{0}
$$

where $\vec{i}_{0}, \bar{j}_{0}, \vec{k}_{0}$ denote the unit vectors of $S R_{0}$.

The equilibrium at rotation is expressed by the differential equations

$$
\left\{\begin{array}{l}
I_{x x}^{j} \frac{d p_{j}}{d t}-\left(I_{y y}^{j}-I_{z z}^{j}\right) q_{j} r_{j}={ }_{\mathrm{ext}}^{x} M_{G^{*}}^{j}, \\
I_{y y}^{j} \frac{d q_{j}}{d t}-\left(I_{z z}^{j}-I_{x x}^{j}\right) r_{j} p_{j}={ }_{\mathrm{ext}}^{y} M_{G^{*}}^{j}, \\
I_{z z}^{j} \frac{d r_{j}}{d t}-\left(I_{x x}^{j}-I_{y y}^{j}\right) p_{j} q_{j}={ }_{\mathrm{ext}}^{z} M_{G^{*}}^{j},
\end{array}\right.
$$

where

$I_{x x}^{j}, I_{y y}^{j}, I_{z z}^{j}$ are the central moments of inertia of the $j$ th body;

$p_{j}, q_{j}, r_{j}$ are the angular velocity components of the $j$ th body expressed in the local frame $S R_{G j}$

${ }_{\text {ext }}^{x} M_{G^{*}}^{j}, \underset{\text { ext }}{y} M_{G^{*}}^{j},{ }_{\text {ext }}^{z} M_{G^{*}}^{j}$ are the Cartesian components of the external resultant moment about the barycenter of the $j$ th body of the external forces expressed in the local frame $S R_{G j}$.

The relations between the angular velocity components expressed in the local frames and the attitude angles and their derivatives are determined by means of the following trans- 
form

$$
\left[\begin{array}{c}
p_{j} \\
q_{j} \\
r_{j}
\end{array}\right]=\left[\begin{array}{ccc}
\cos \alpha_{x}^{j} \cos \alpha_{z}^{j} & 0 & \sin \alpha_{z}^{j} \\
\sin \alpha_{x}^{j} & 1 & 0 \\
-\cos \alpha_{x}^{j} \sin \alpha_{z}^{j} & 0 & \cos \alpha_{z}^{j}
\end{array}\right] \cdot\left[\begin{array}{c}
\dot{\alpha}_{x}^{j} \\
\dot{\alpha}_{y}^{j} \\
\dot{\alpha}_{z}^{j}
\end{array}\right] .
$$

The $j$ th body angular velocity components, expressed in the absolute frame $S R_{0}$, are obtained by using the transform matrix (1).

\section{Numerical results}

The numerical results discussed in this section regard:

- Code validation;

- Monitoring the time-steps variation under strongly nonstationary conditions;

- Investigation on some instability phenomena.

\subsection{Code validation}

In order to validate the developed code, a comparison is made with the results supplied by a commercial multibody software: in particular, the analyses concern the behavior of a single wheelset and of a complete bogie behavior under stable, critical and unstable conditions.

To obtain the critical speed of the wheelset and the bogie-namely $V_{\mathrm{cr}}^{w}$ and $V_{\mathrm{cr}}^{b}$-moving on a straight path, the two systems - the wheelset and the bogie-running with a longitudinal velocity $V_{\text {long }}$ are initially perturbed with a transverse velocity component $V_{\text {trans }}$. In all simulations, the $V_{\text {trans }}$ is set equal to $0.1 \mathrm{~m} / \mathrm{s}$.

For the wheelset case, let us assume that $V_{\text {long }}$ is lower than the $V_{\mathrm{cr}}^{w}$. For establishing the critical velocities, the hunting motion after the initial kinematic perturbation should be monitored. If the amplitudes of the oscillations decrease with time, then $V_{\text {long }}<V_{\mathrm{cr}}^{w}$, and it is necessary to increment $V_{\text {long }}$. Otherwise, if the amplitudes increase, then $V_{\text {long }}>V_{\mathrm{cr}}^{w}$, and $V_{\text {long }}$ must be decreased. When the amplitudes are almost constant in time, the condition for which $V_{\text {long }}=V_{\mathrm{cr}}^{w}$ is given.

The same procedure is applied to the computation of $V_{\mathrm{cr}}^{b}$. In this case, kinematic perturbations are initially applied to both wheelsets.

Figures 15, 16 and 17 show the transverse displacement, the yaw angle of the wheelset and the transverse tangent contribution of the tangent component acting on the two wheels in three different initial conditions, that is, $V_{\text {long }}<V_{\mathrm{cr}}^{w}, V_{\text {long }}=V_{\mathrm{cr}}^{w}$ and $V_{\text {long }}>V_{\mathrm{cr}}^{w}$.

Figures 18, 19, 20 and 21 show the transverse displacement and the yaw angle of the three masses of the bogie - the front and back wheelsets and the bogie frame-and the two contributions of the tangent component acting on the four wheels in the case of $V_{\text {long }}<$ $V_{\mathrm{cr}}^{w}$. The symbols in the figures have the following meaning: $\mathrm{W}=$ wheel, $\mathrm{WS}=$ wheelset, $\mathrm{CMS}=$ commercial multibody software.

The mentioned figures demonstrate the agreement between our model and those developed within the commercial multibody software. This is observed for both the displacements (kinematics) and the contact forces (dynamics). Moreover, we highlight that the displacement and the yaw angle present a very similar periodicity; this proves that the hunting motion is coupled and thus generated by the interaction of the transverse displacement and the yaw rotation. From Figs. 18 and 19, one observe that all the masses concerning the bogie 


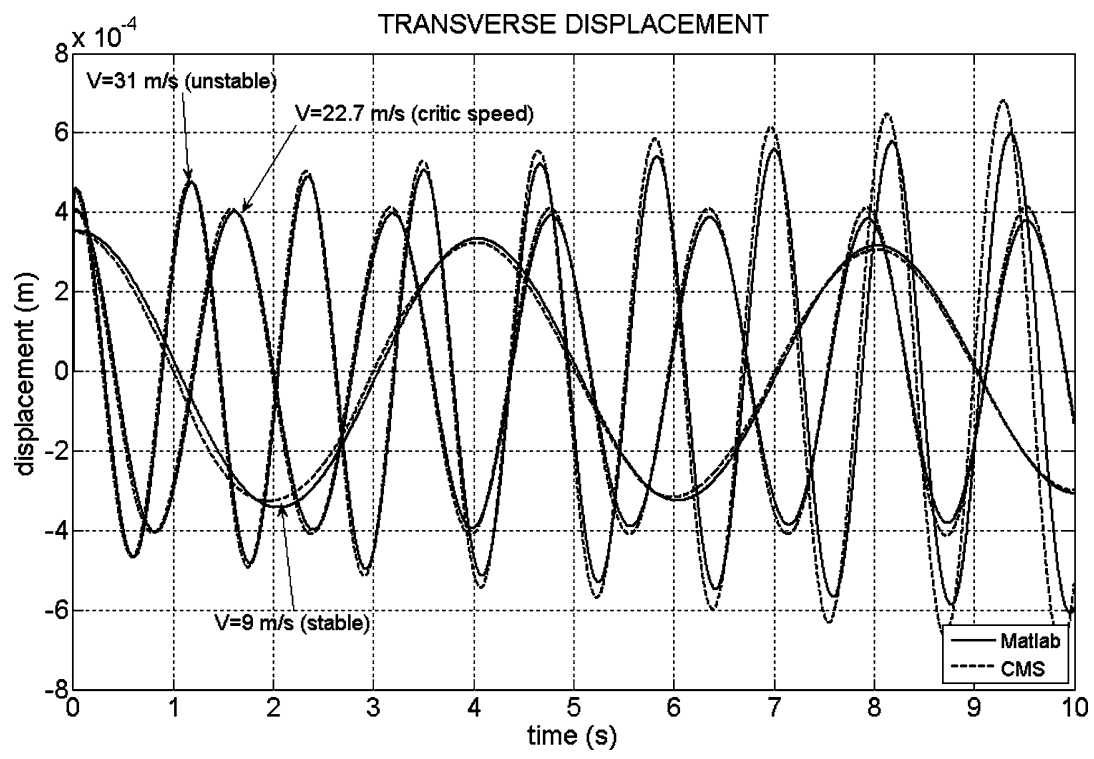

Fig. 15 Plots of the transverse displacement of the wheelsets obtained by means of the Matlab software and the commercial multibody software with longitudinal velocities corresponding to stable, critical and unstable conditions

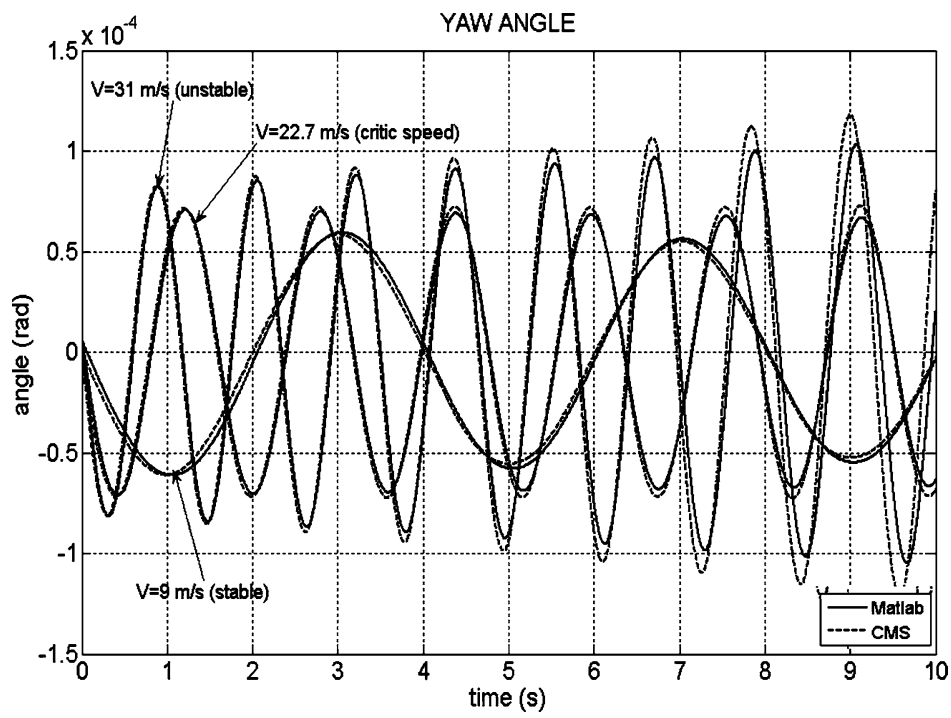

Fig. 16 Plots of the yaw angle of the wheelsets obtained by means of the Matlab software and the commercial multibody software with longitudinal velocities corresponding to stable, critical and unstable conditions

oscillate in phase; this behavior is characteristic of the rectilinear track when the perturbations are applied to the front and back wheelsets at the same manner. On the contrary, in curved tracks one can observe that the two wheelsets yaw angles oscillate in counter-phase changing the hunting mechanism involved [20]. 


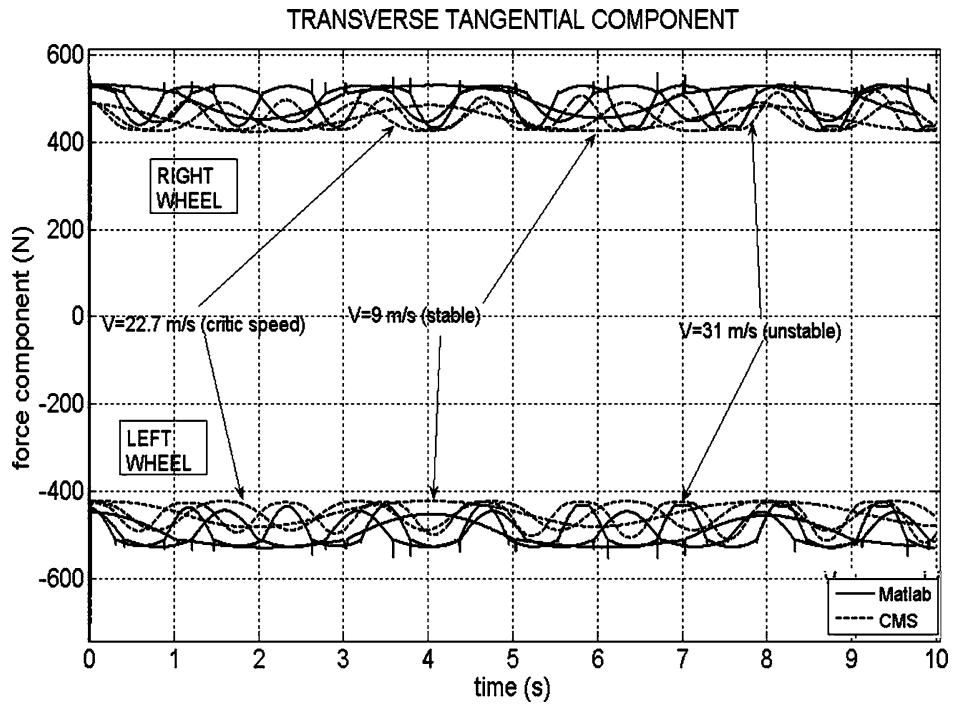

Fig. 17 Plots of the transverse tangent component acting on the two wheels obtained by means of the Matlab software and the commercial multibody software with longitudinal velocities corresponding to stable, critical and unstable conditions

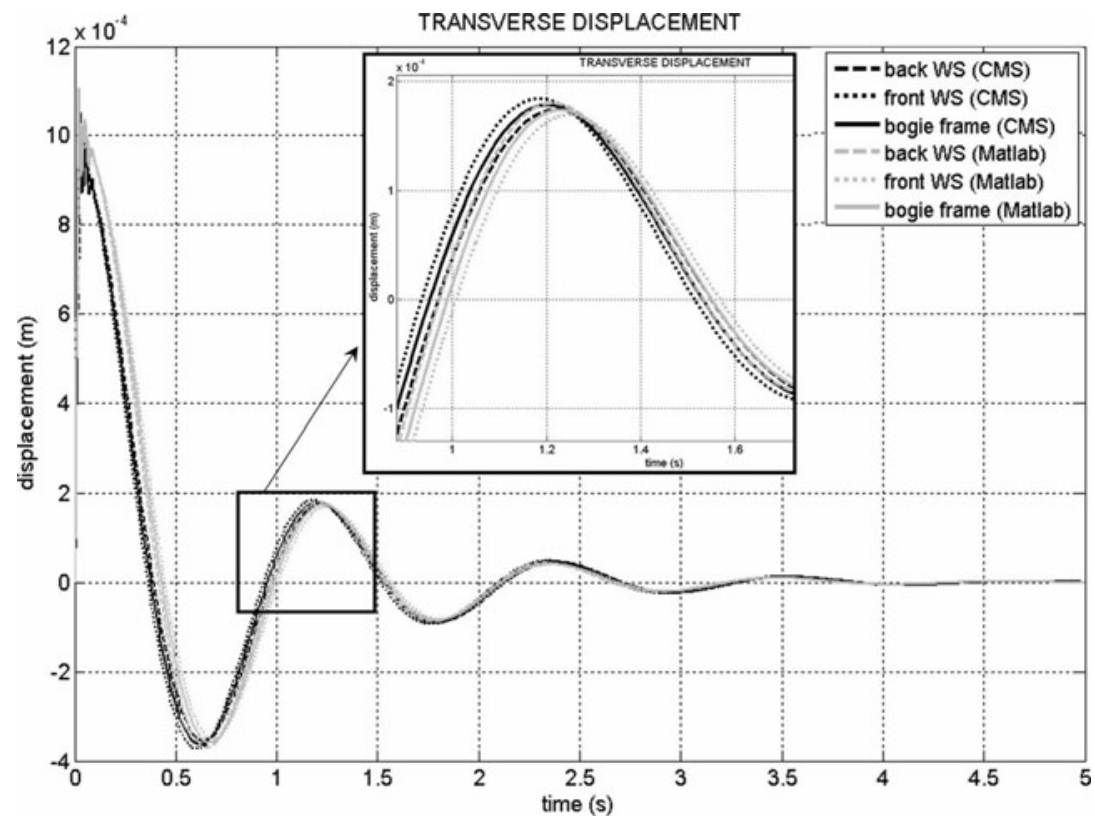

Fig. 18 Plots of the transverse displacement of the two wheelsets and the bogie frame obtained by means of the Matlab software and the commercial multibody software

\subsection{Time-steps monitoring}

We observed that the required step strongly depends on the degree of variation of the variables that control the motion of the system. When the variables are stationary, the integration 


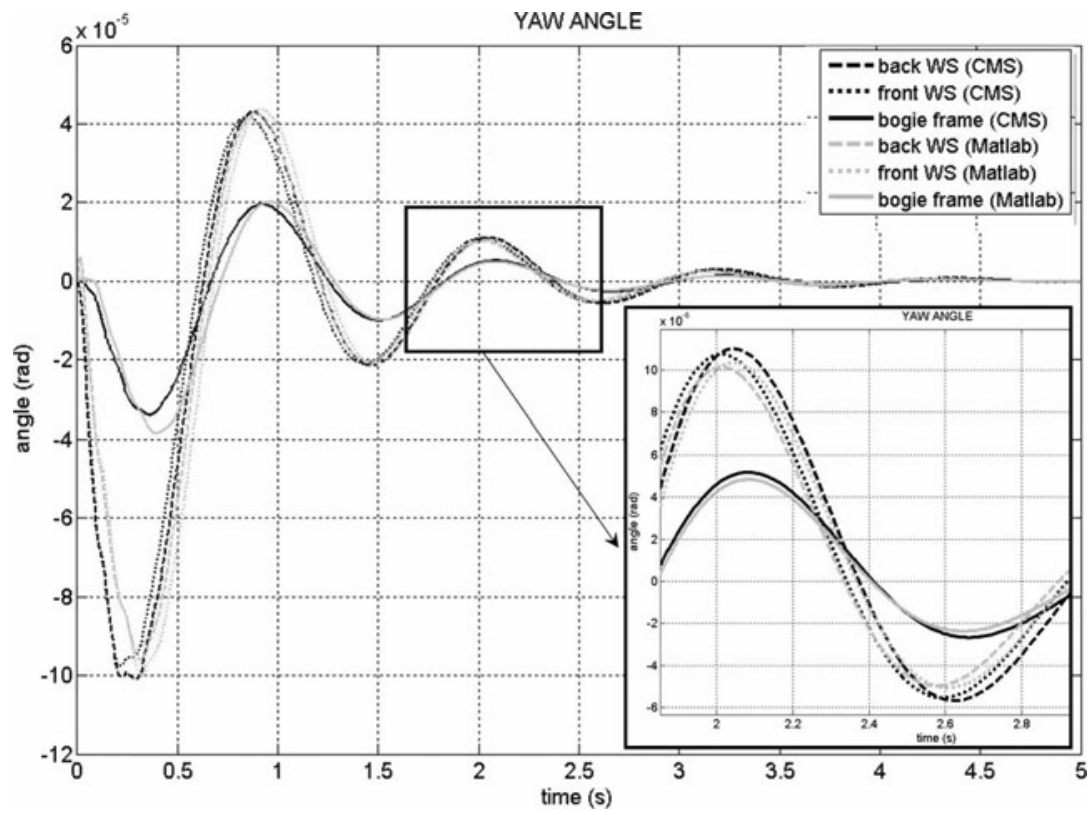

Fig. 19 Plots of the yaw angle of the two wheelsets and the bogie frame obtained by means of the Matlab software and the commercial multibody software

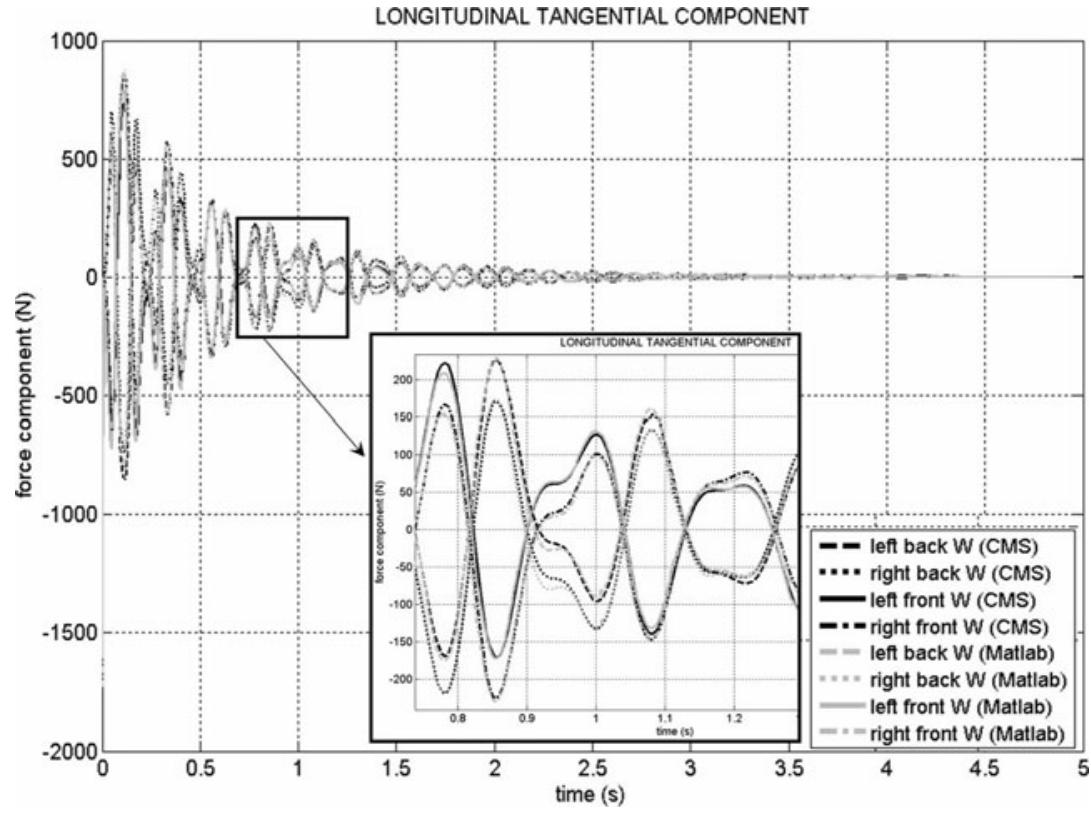

Fig. 20 Plots of the longitudinal tangent components acting on the four wheels obtained by means of the Matlab software and the commercial multibody software 


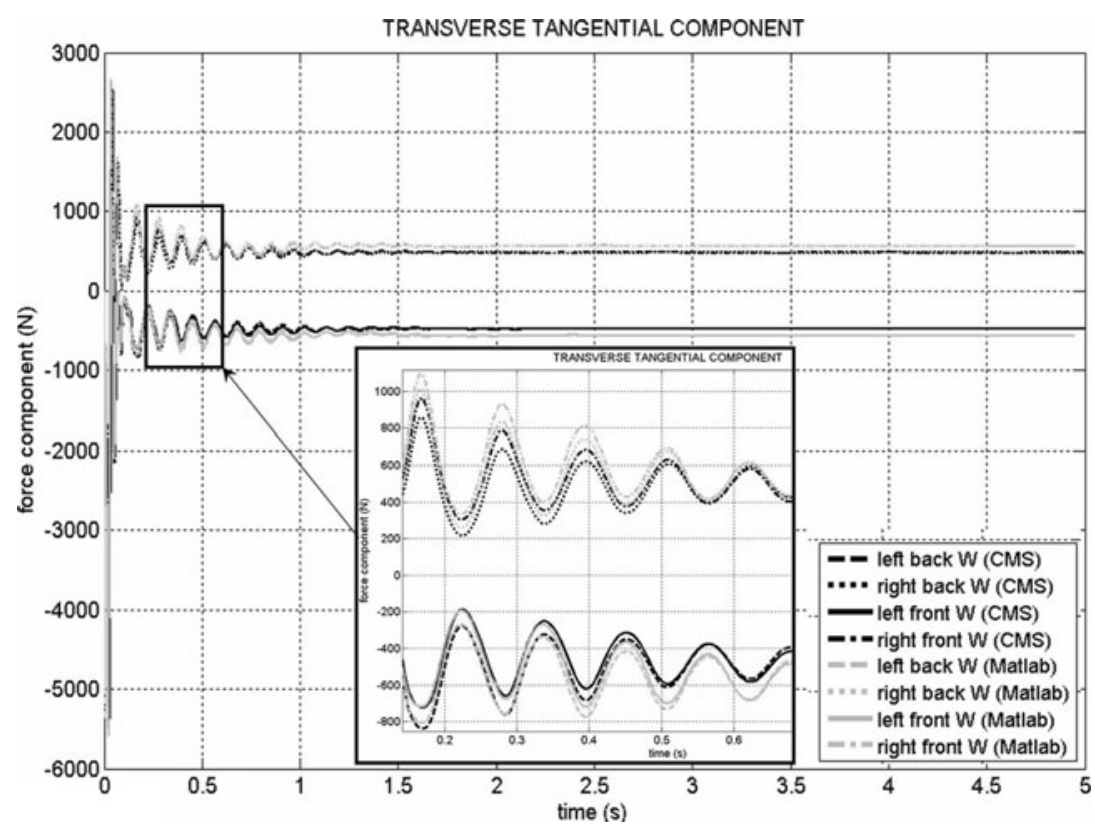

Fig. 21 Plots of the transverse tangent component acting on the four wheels obtained by means of the Matlab software and the commercial multibody software

step increases continuously; on the contrary, when many perturbations occur, for example, when the contact point crosses from the wheel tread to the flange, the step attains its lower values.

Figure 22 compares in a logarithmic scale three different simulations of the same system having equal time span but with the following initial conditions:

- In absence of perturbation (Fig. 22(a));

- With initial transverse perturbation set to $0.1 \mathrm{~m} / \mathrm{s}$, so that the contact points remain on the wheel tread (Fig. 22(b));

- With initial transverse perturbation set to $0.35 \mathrm{~m} / \mathrm{s}$ in such a way that the contact points concern both the tread and the flange wheel (Fig. 22(c)).

One can observe that:

(a) The step lengths increase with time up to $0.25 \mathrm{~s}$ in the case of unperturbed motion; the sharp decrease observed after the first peak is due to the vertical motion of the wheelset in the transient phase;

(b) In the case of small initial perturbation, for instance $0.1 \mathrm{~m} / \mathrm{s}$, we have a hunting motion and the step length, after a first increasing phase, stabilizes on a value close to 1.2 . $10^{-3} \mathrm{~s}$. This behavior can be justified considering the higher variation of the motion variables by respect to the unperturbed motion;

(c) With a perturbation causing an additional contact at the flange, the rate of change of contact features is very high and the contact points switches between the tread and the flange continuously. Hence, due to the sharp variations, the integration step is reduced.

The step length influences the duration of the simulation and the global step number. In fact, we recorded 125, 320 and 4900 steps in the first, second and last case, respectively. 
Fig. 22 Step length in logarithmic scale in three different cases: (a) unperturbed motion; (b) small initial perturbation without flange contact; (c) relevant initial perturbation with flange contact

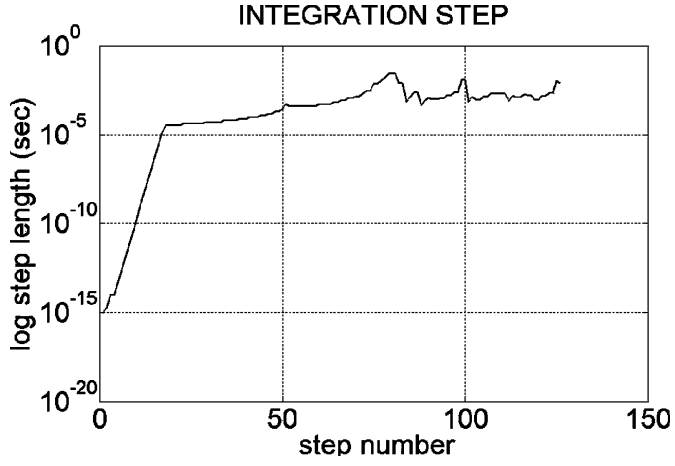

(a)

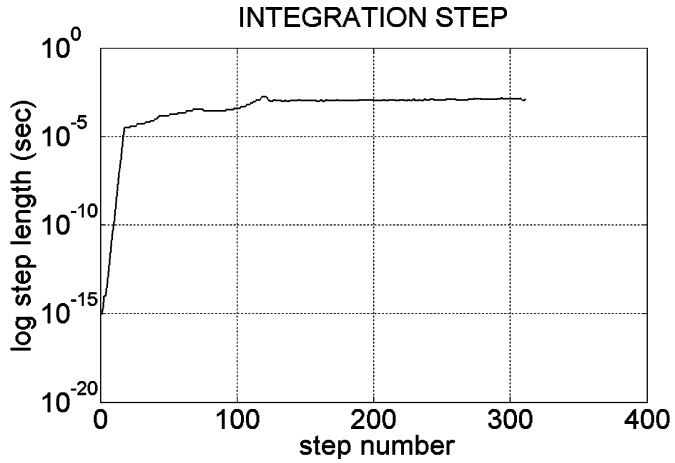

(b)

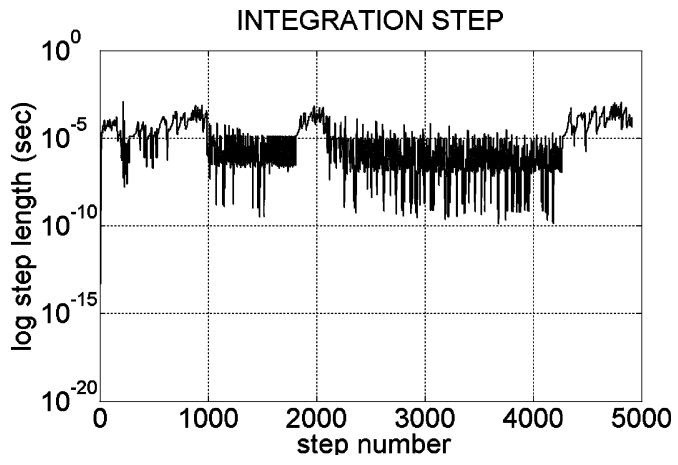

(c)

\subsection{The critical speed}

It is well known that the critical speed of a wheelset is affected by the external load applied on it. In fact, one can plot (see Fig. 23(a)) the critical speed versus the wheelset load ratio, defined as the ratio between the vertical load directly applied on the wheelset barycenter and the wheelset weight. At the same time one can monitor the frequency of the hunting by means of a Fourier analysis of the displacement versus the load ratio (see Fig. 23(b)). Figure 24(a) shows the bogie critical speed versus the bogie load ratio defined as the ratio 
Fig. 23 Plots of the critical speed and hunting frequency of the wheelset as regards the wheelset load ratio

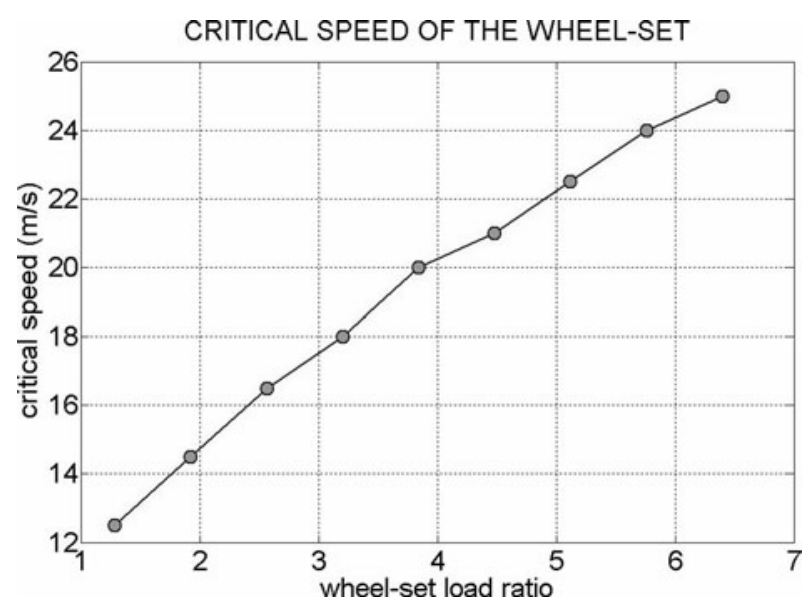

(a)

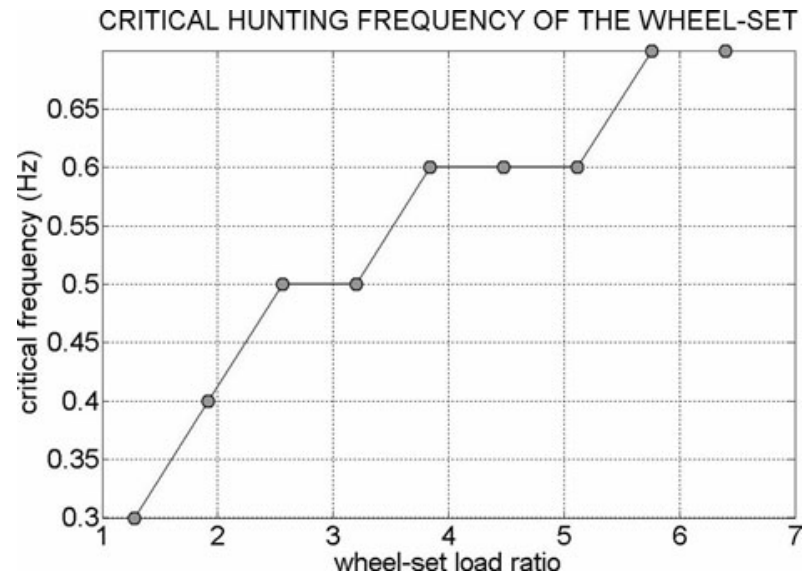

(b)

between the vertical load directly applied on the frame bogie and its weight. Figure 24(b) reports the bogie hunting frequency versus the bogie load ratio.

As one can observe from the figures, the critical bogie speed is much higher when compared to the wheelset one with the same load ratio. This is mainly due:

- The presence of spaced wheelsets on the bogie;

- The presence of the primary suspension system.

The strongly perturbed wheelset is not able, by means of the contact force actions, to generate a set of forces able to damp the motion. However, the stabilizing action generated by the bogie can reach very high values as it is promoted by the distance of the two wheelset.

Also the primary suspension system contributes to the stabilizing action.

Another interesting evidence is the increment of the critical speed when the load ratio increases. This phenomenon reveals the importance of the contact forces on the stability of the system. In fact, when the external load is increased, also the normal and tangential components rise accordingly. 
Fig. 24 Plots of the critical speed and hunting frequency of the bogie vs. the bogie load ratio

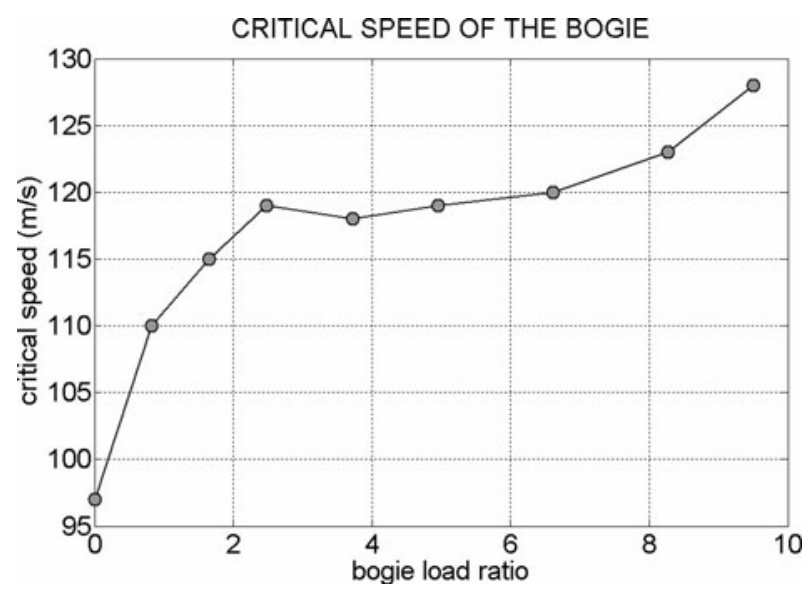

(a)

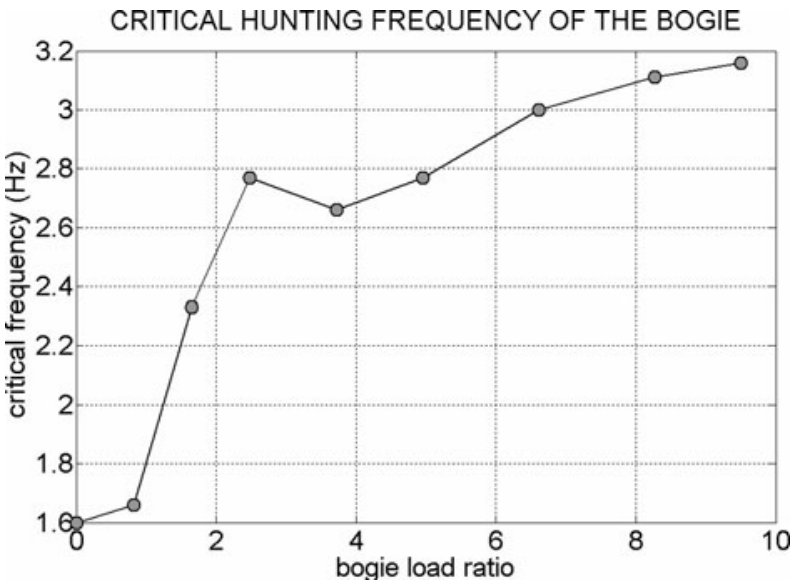

(b)

The system has better possibilities of reacting to the perturbations. In order to explain this concept, we can imagine the limit case where all the external loads, included the weight, are null. The contact forces are null as well and the system cannot counter any perturbation.

We recorded a different critical speed variation between wheelset and bogie. For the latter, at low load-ratio values the critical speed gradient is high. However, with reference to Fig. 24(a), when the load-ratio reaches more realistic values, the critical speed keeps almost constant.

The realistic load-ratio ranges from 3 , for an empty wagon, to 8, for a fully loaded one. Within these limits, the critical speed is almost constant. Nevertheless, the suspension system parameters have a strong influence on the extension of the stationary region not herein discussed.

When considering the hunting frequencies (Fig. 24(b)), their variation is strongly affected by the load ratio. However, gradients are higher at low load ratio values for both the wheelset and the bogie.

It should point out that such critical conditions can be approached also when an emergency braking is necessary. As a matter of fact, in an emergency braking of a long train, 
some wagons can have their actual load-ratio reduced due to the friction forces transmitted by bumpers.

A final consideration regards the comparison between hunting in a rectilinear or a curved track. Apparently, hunting is strongly influenced by the radius of curvature of the track [20]. However, if one examines the rectilinear case after a severe initial perturbation, until lateral flange contact is involved, the critical speed decreases significantly, reducing its value from 123 to $43 \mathrm{~m} / \mathrm{s}$, much closer to the values computed for curved tracks $(32 \mathrm{~m} / \mathrm{s}$ for an $88 \mathrm{~m}$ radius track). This comparison suggests the idea that the difference is mainly centered on the perturbation magnitude rather than on the track radius of curvature.

\section{Conclusions}

In the paper, the model and dynamic behavior of bogies composed of two wheelsets and a frame is presented. The computer simulation includes a wheel-rail contact that accounts for a different local stiffness of tread and flange wheel. Due to optimized use of a preliminary compiled look-up table, the computation time is reduced considerably. In particular, all data that define the complex contact between wheel and the rail are stored as a function of two independent variables.

Both straight and curved railways could be analyzed for the monitoring of the hunting phenomenon.

Because of its influence on overall cpu-time, the interpolation procedure of the look-up table has been optimized.

A particular interest is given by the results concerning hunting instability when varying the load on each axis. A different influence of the load ratio on the critical speed of the wheelset and of the bogie assembly has been recorded. For the wheelset, the load ratio has an almost linearly proportional influence. For the bogie, this influence is much more limited within the range of practical interest.

The software developed was validated through the comparison with the results obtained by a commercial software.

\section{References}

1. Shabana, A.A., Zaazaa, K.E., Escalona, J.L., Sany, J.R.: Development of elastic force model for wheelrail contact problems. J. Sound Vib. 269, 295-325 (2004)

2. Polach, O.: On non-linear methods of bogie stability assessment using computer simulations. IMechE J. Rail Rapid Transit 220(F), 13-27 (2006)

3. Parena, D., Kuka, N., Vivalda, P., Kik, W.: Stability investigation and narrow curving analysis of a streetcar model. In: 4th Adams/Rail Users' Conference, Uthrect (1999)

4. Pombo, J., Ambrosio, J.A.C.: General spatial curve joint for rail guided vehicles: kinematics and dynamics. Multibody Syst. Dyn. 9, 237-264 (2003)

5. Bozzone, M., Pennestrì, E., Salvini, P.: A compliance based method for wheel-rail contact analysis. In: International Conference on Contact Mechanics and Wear of Rail/Wheel Systems, Florence, 15-18 Sept. (2009)

6. Kalker, J.J.: Three-dimensional Elastic Bodies in Rolling Contact. Kluwer Academic, Dordrecht (1990)

7. Kalker, J.J.: Rolling contact phenomena. In: Jacobson, B., Kalker, J.J. (eds.) International Centre for Mechanical Sciences. CISM Courses and Lectures, vol. 411. Springer, Berlin (2000)

8. Lee, S.Y., Cheng, Y.C.: Hunting stability analysis of high-speed railway vehicle bogies on tangent tracks. J. Sound Vib. 282, 881-898 (2005)

9. Meli, E., Auciello, J., Malvezzi, M., Papini, S., Pugi, L., Rindi, A.: Determination of wheel rail contact points with semi analytic methods. Multibody Syst. Dyn. 20, 327-358 (2008) 
10. Bosso, N., Gugliotta, A., Somà, A.: Realizzazione di un modulo di contatto a ruote indipendenti. In: XXXI Aias Conference, settembre, Parma (2002) (in Italian)

11. Kalker, J.J.: A fast algorithm for the simplified theory of rolling contact. Veh. Syst. Dyn. 11, 1-13 (1982)

12. Polach, O.: Influence of locomotive tractive effort on the forces between wheel and rail. Veh. Syst. Dyn. Suppl. 35, 7-22 (2001)

13. Vermeulen, P.J., Johnson, K.L.: Contact of non-spherical bodies transmitting tangential forces. ASME J. Appl. Mech. 31, 338-340 (1964)

14. True, H.: Recent advances in the fundamental understanding of railway vehicle dynamics. Int. J. Veh. Des. 40(1/2/3), 251-264 (2006)

15. Banerjee, N., Karmakar, R.: Bond graph modelling of rail wheelset on curved track. Simulation 83(10), 695-706 (2007)

16. Gollasch, G., Gökhan, G., Meisinger, R., Chen, W.-G., Shu, G.-W.: Simulation of railway-bogie. ISSN 1616-0726 Sonderdruck Schriftenreihe der Georg-Simon-Ohm-Fachhochschule Nürnberg Num. 3, Nürnberg, 2001

17. Huang, Y.M., Chen, K.L., Wang, T.S.: Running stability simulation and field testing of two axle-bogie design. Int. J. Veh. Des. 26(2/3), 131-145 (2001)

18. Kortum, W., Goodall, R.M.: Mechatronic developments for railway vehicles of the future. Control Eng. Pract. 10, 887-898 (2002)

19. Pearson, J.T., Goodall, R.M., Mei, T.X., Shen, S., Kossmann, C., Polach, O., Himmelstein, G.: Design and experimental implementation of an active stability system for a high speed bogie. In: Extensive Summaries of the 18th IAVSD Symposium Dynamics of Vehicle on Roads and Tracks, Atsugi, Kanagawa, Japan, August 24-30 (2003)

20. Bozzone, M., Pennestrì, E., Salvini, P.: Behaviour analysis of a conventional bogie and optimization of its suspension system at stability limit. In: 1st Joint Int. Conference on Multibody System Dynamics, Lappeenranta, Finland, May 25-27 (2010) 